\title{
Modifying surfaces in 4-manifolds by twist spinning
}

\author{
HEE JUNG KIM
}

\begin{abstract}
In this paper, given a knot $K$, for any integer $m$ we construct a new surface $\Sigma_{K}(m)$ from a smoothly embedded surface $\Sigma$ in a smooth 4-manifold $X$ by performing a surgery on $\Sigma$. This surgery is based on a modification of the 'rim surgery' which was introduced by Fintushel and Stern, by doing additional twist spinning. We investigate the diffeomorphism type and the homeomorphism type of $(X, \Sigma)$ after the surgery. One of the main results is that for certain pairs $(X, \Sigma)$, the smooth type of $\Sigma_{K}(m)$ can be easily distinguished by the Alexander polynomial of the knot $K$ and the homeomorphism type depends on the number of twist and the knot. In particular, we get new examples of knotted surfaces in $\mathbb{C} \mathbf{P}^{2}$, not isotopic to complex curves, but which are topologically unknotted.
\end{abstract}

57R57; 14J80, 57R95

\section{Introduction}

Let $X$ be a smooth 4 -manifold and $\Sigma$ be an embedded positive genus surface and nonnegative self-intersection. In [3], Fintushel and Stern introduced a technique, called 'rim surgery', of modifying $\Sigma$ without changing the ambient space $X$. This surgery on $\Sigma$ may change the diffeomorphism type of the embedding $\Sigma_{K}$ but the topological embedding is preserved when $\pi_{1}(X-\Sigma)$ is trivial. Rim surgery is determined by a knotted arc $K_{+} \in B^{3}$, and may be described as follows. Choose a curve $\alpha$ in $\Sigma$, which has a neighborhood $S^{1} \times B^{3}$ meeting $\Sigma$ on an annulus $S^{1} \times I$. Replacing the pair $\left(S^{1} \times B^{3}, S^{1} \times I\right)$ by $\left(S^{1} \times B^{3}, S^{1} \times K_{+}\right)$gives a new surface $\Sigma_{K}$ in $X$.

In [17], Zeeman described the process of twist-spinning an $n$-knot to obtain an $(n+1)-$ knot. Here an $n-k n o t$ is a locally flat pair $\left(S^{n+2}, K\right)$ with $K \cong S^{n}$. Then here is the description for the process of twist-spinning to obtain a knot in dimension 4: Suppose we have a knotted arc $K_{+}$in the half 3-space $\mathbb{R}_{+}^{3}$, with its end points in $\mathbb{R}^{2}=\partial \mathbb{R}_{+}^{3}$. Spinning $\mathbb{R}_{+}^{3}$ about $\mathbb{R}^{2}$ generates $\mathbb{R}^{4}$, the arc $K_{+}$generates a knotted 2-sphere in $\mathbb{R}^{4}$, called a spun knot. During the spinning process we spin the arc $K_{+} m$ times keeping its end points within $\mathbb{R}_{+}^{3}$, obtaining again a 2 -sphere $K(m)$ in $\mathbb{R}^{4}$. A more explicit definition is the following. 
For any $1-$ knot $\left(S^{3}, K\right)$, let $\left(B^{3}, K_{+}\right)$be its ball pair with the knotted arc $K_{+}$. Let $\tau$ be the diffeomorphism of $\left(B^{3}, K_{+}\right)$, called 'twist map' defined in Section 2. Then for any integer $m$ this induces a 2-knot called the $m$-twist spun knot

$$
\left(S^{4}, K(m)\right)=\partial\left(B^{3}, K_{+}\right) \times B^{2} \cup_{\partial}\left(B^{3}, K_{+}\right) \times_{\tau^{m}} \partial B^{2}
$$

where $\left(B^{3}, K_{+}\right) \times_{\tau^{m}} \partial B^{2}$ means that $\left(B^{3}, K_{+}\right) \times[0,1] /(x, 0)=\left(\tau^{m} x, 1\right)$.

In this paper, using these two ideas — rim surgery and spun knot - we will construct a new surface, denoted by $\Sigma_{K}(m)$, from the embedded surface in $X$ without changing its ambient space. Our technique may be called a 'twist rim surgery'. We will see later (in Section 3 and Section 4) that the smooth and topological type of $\Sigma_{K}(m)$ obtained by twist rim surgery depends on $m, K$, and $\Sigma$. For a precise definition of the surgery, we will give two descriptions of $\Sigma_{K}(m)$. One is provided by using the twist map $\tau$ in the construction of Zeeman's twist spun knot. The other one can be obtained by performing the same operation which Fintushel and Stern introduced in [4] as it corresponds to doing a surgery on a homologically essential torus in $X$. In [4], they constructed exotic manifolds $X_{K}$ according to a knot $K$ and also showed that the Alexander polynomial $\Delta_{K}(t)$ of $K$ can detect the smooth type of $X_{K}$.

In our circumstance, we consider a pair $(X, \Sigma)$, where $X$ is a smooth simply connected 4-manifold and $\Sigma$ is an embedded genus $g$ surface with self-intersection $n \geq 0$ such that the homology class $[\Sigma]=d \cdot \beta$, where $\beta$ is a primitive element in $H_{2}(X)$ and $\pi_{1}(X-\Sigma)=\mathbb{Z} / d$. Then in Section 3, we will study the smooth type of $\Sigma_{K}(m)$ obtained by performing twist rim surgery on $\Sigma$. In fact, using the result in [3], we conclude that the Alexander polynomial $\Delta_{K}(t)$ of $K$ can distinguish the smooth type of $\Sigma_{K}(m)$. In particular, applying this result to $\mathbb{C} \mathbf{P}^{2}$ we can get new examples of knotted surfaces in $\mathbb{C} \mathbf{P}^{2}$, not isotopic to complex curves. This solves, for an algebraic curve of degree $\geq 3$, Problem 4.110 in the Kirby list [9]. Note that $d=1,2$ which are the only degrees where the curve is a sphere, are still open.

In Section 4, we will study topological conditions under which $\left(X, \Sigma_{K}(m)\right)$ is pairwise homeomorphic to $(X, \Sigma)$. This problem is also related to the knot type of $K$ and the relation between $d$ and $m$. In particular, if $d \not \equiv \pm 1(\bmod m)$ then computing the fundamental group of the exterior of surfaces in $X$ we easily distinguish $\left(X, \Sigma_{K}(m)\right)$ and $(X, \Sigma)$ for some nontrivial knot $K$. But when $d \equiv \pm 1(\bmod m)$, it turns out that the fundamental group $\pi_{1}\left(X-\Sigma_{K}(m)\right)$ is same as $\pi_{1}(X-\Sigma)=\mathbb{Z} / d$. So, in the case $d \equiv \pm 1(\bmod m)$ we show that if $K$ is a ribbon knot and the $d$-fold cover of the knot complement $S^{3}-K$ is a homology circle then $(X, \Sigma)$ and $\left(X, \Sigma_{K}(m)\right)$ are topologically equivalent. This means that there is a pairwise homeomorphism $(X, \Sigma) \longrightarrow\left(X, \Sigma_{K}(m)\right)$. 


\section{Definitions}

Let $X$ be a smooth 4-manifold and let $\Sigma$ be an embedded surface of positive genus $g$. Given a knot $K$ in $S^{3}$, let $E(K)$ be the exterior $\operatorname{cl}\left(S^{3}-K \times D^{2}\right)$ of $K$. First we need to consider a certain diffeomorphism $\tau$ on $\left(S^{3}, K\right)$ which will be used to define our surgery. Take a tubular neighborhood of the knot and then using a suitable trivialization with 0-framing, let $\partial E(K) \times I=K \times \partial D^{2} \times I$ be a collar of $\partial E(K)$ in $E(K)$ with $\partial E(K)$ identified with $\partial E(K) \times\{0\}$. Define $\tau:\left(S^{3}, K\right) \longrightarrow\left(S^{3}, K\right)$ by

$$
\tau\left(x \times e^{i \theta} \times t\right)=x \times e^{i(\theta+2 \pi t)} \times t \text { for } x \times e^{i \theta} \times t \in K \times \partial D^{2} \times I
$$

and $\tau(y)=y$ for $y \notin K \times \partial D^{2} \times I$.

Note that $\tau$ is not the identity on the collar $\partial E(K) \times I=K \times \partial D^{2} \times I$. However, it is the identity on the exterior $\operatorname{cl}\left(S^{3}-K \times \partial D^{2} \times I\right)$ of the collar. If we restrict $\tau$ to the exterior of the knot $K$ then $\tau$ is isotopic to the identity although the isotopy is not the identity on the boundary of the knot complement. Explicitly, the isotopy can be given as the following. For any $s \in[0,1]$,

$$
\tau_{s}\left(x \times e^{i \theta} \times t\right)=x \times e^{i \theta+2 \pi t(1-s)+2 \pi s} \times t .
$$

We will refer to this diffeomorphism $\tau$ as a twist map.

Now take a non-separating curve $\alpha$ in $\Sigma$. Then choose a trivialization of the normal bundle $\left.v(\Sigma)\right|_{\alpha}$ in $X, \alpha \times I \times D^{2}=\alpha \times\left. B^{3} \longrightarrow v(\Sigma)\right|_{\alpha}$ where $\alpha \times I$ corresponds to the normal bundle $v(\alpha)$ in $\Sigma$. For any trivialization of the tubular neighborhood of $\alpha$ we can construct a new surface from $\Sigma$ using the chosen curve. We will choose a specific framing of $\alpha$ later in Section 3 to study the diffeomorphism type of the new surface constructed in the way discussed now. Identifying $\alpha$ with $S^{1}$, two descriptions of the construction of $\left(X, \Sigma_{K}(m)\right)$ called $m$-twist rim surgery follow.

Definition 2.1 Define for any integer $m$,

$$
\left(X, \Sigma_{K}(m)\right)=(X, \Sigma)-S^{1} \times\left(B^{3}, I\right) \cup_{\partial} S^{1} \times_{\tau^{m}}\left(B^{3}, K_{+}\right) .
$$

Note that for $m=0, \Sigma_{K}(m)$ is the surface obtained by rim surgery. In [3], its smooth type was studied when $\pi_{1}(X-\Sigma)$ is trivial. As in the paper [3], we will consider the smooth type of the new surface obtained by $m$-twist surgery in the extended case where $\pi_{1}(X-\Sigma)$ is cyclic.

If $\alpha$ is a trivial curve, that is it bounds a disk in $\Sigma$, we can simply write $\left(X, \Sigma_{K}(m)\right)$ as the following. 
Lemma 2.2 If $\alpha$ is a trivial curve in $\Sigma$, then $\left(X, \Sigma_{K}(m)\right)$ is the connected sum $(X, \Sigma)$ with the $m$-twist spun knot $\left(S^{4}, K(m)\right)$ of $\left(S^{3}, K\right)$.

Proof Considering the decomposition of $\left(X, \Sigma_{K}(m)\right)$ in Definition 2.1.

$$
\left(X, \Sigma_{K}(m)\right)=(X, \Sigma)-S^{1} \times\left(B^{3}, I\right) \cup_{\partial} S^{1} \times_{\tau^{m}}\left(B^{3}, K_{+}\right),
$$

we write the boundary of the ball $\left(B^{3}, I\right)$ in the definition as

$$
\partial\left(B^{3}, I\right)=\left(S^{2},\{N, S\}\right)=\left(D_{+}^{2},\{N\}\right) \cup\left(D_{-}^{2},\{S\}\right)
$$

where $D_{+}^{2}, D_{-}^{2}$ are 2-disks and N, S are north and south poles respectively. Also recall that we identified $\alpha$ as $S^{1}$ in the definition and by the choice of $\alpha$, let's denote the disk bounded by $\alpha$ as $B^{2}$ in $\Sigma$. Then we can rewrite

$$
\begin{aligned}
& \left(X, \Sigma_{K}(m)\right)= \\
& \left((X, \Sigma)-\left(S^{1} \times\left(B^{3}, I\right) \cup B^{2} \times\left(D_{+}^{2},\{N\}\right)\right)\right) \cup\left(B^{2} \times\left(D_{+}^{2},\{N\}\right) \cup S^{1} \times \tau^{m}\left(B^{3}, K_{+}\right)\right) .
\end{aligned}
$$

Note that the first component of this decomposition is

$$
(X, \Sigma)-S^{1} \times\left(B^{3}, I\right) \cup_{\partial B^{2} \times D_{+}^{2}} B^{2} \times\left(D_{+}^{2},\{N\}\right)=(X, \Sigma)-\left(B^{4}, B^{2}\right) .
$$

In the second component

$$
B^{2} \times\left(D_{+}^{2},\{N\}\right) \cup_{\partial B^{2} \times D_{+}^{2}} S^{1} \times{ }_{\tau^{m}}\left(B^{3}, K_{+}\right),
$$

gluing $B^{2} \times\left(D_{-}^{2},\{S\}\right)$ to $B^{2} \times\left(D_{+}^{2},\{N\}\right)$ along $B^{2} \times \partial D_{+}^{2}$ and then taking it out later again we can write

$$
\begin{array}{r}
\left(B^{2} \times\left(D_{+}^{2},\{N\}\right)\right) \cup_{B^{2} \times \partial D_{+}^{2}}\left(B^{2} \times\left(D_{-}^{2},\{S\}\right)\right) \cup_{\partial}\left(S^{1} \times \tau^{m}\left(B^{3}, K_{+}\right)\right)-\left(B^{2} \times\left(D_{-}^{2},\{S\}\right)\right) \\
=\left(B^{2} \times \partial\left(B^{3}, K_{+}\right)\right) \cup_{\partial}\left(S^{1} \times \tau^{m}\left(B^{3}, K_{+}\right)\right)-\left(B^{2} \times\left(D_{-}^{2},\{S\}\right)\right) .
\end{array}
$$

Considering the definition of twist spun knot in Section 1 we can realize this is

$$
\left(S^{4}, K(m)\right)-\left(B^{2} \times\left(D_{-}^{2},\{S\}\right)\right) .
$$

So,

$$
\left(X, \Sigma_{K}(m)\right)=\left((X, \Sigma)-\left(B^{4}, B^{2}\right)\right) \cup\left(\left(S^{4}, K(m)\right)-B^{2} \times\left(D_{-}^{2},\{S\}\right)\right)
$$

where the union is taken along the boundary.

Let's move on to another description of $\left(X, \Sigma_{K}(m)\right)$ which is useful in distinguishing the diffeomorphism types of $\Sigma_{K}(m)$. For a non-separating curve $\alpha$ in $\Sigma$, after a trivialization, the normal bundle $\alpha$ in $X$ is of the form $\alpha \times I \times D^{2}=\alpha \times B^{3}$ where 
$\alpha \times I$ in $\Sigma$. Consider $\alpha \times \gamma \subset \alpha \times I \times D^{2}$ where $\gamma$ is a pushed-in copy of the meridian circle $\{0\} \times \partial D^{2} \subset I \times D^{2}$. Under our trivialization, $\alpha \times \gamma$ is diffeomorphic to a torus $T$ in $X-\Sigma$, called a rim torus by Fintushel and Stern. Note that this torus $T$ is nullhomologous in $X$. Let $N(\gamma)$ be a tubular neighborhood of $\gamma$ in $B^{3}=I \times D^{2}$ and $\gamma^{\prime}$ be the curve $\gamma$ pushed off into $\partial N(\gamma)$. Then we will identify $\alpha \times N(\gamma)$ as a neighborhood $N(T)$ of $T$ under the trivialization so that $\alpha \times\left. N(\gamma) \subset v(\Sigma)\right|_{\alpha} \subset v(\Sigma)$. For a knot $K$ in $S^{3}$, let's denote by $\mu_{K}$ the meridian and $\lambda_{K}$ the longitude of the knot. Now consider the following manifold

$$
\alpha \times\left(B^{3}-N(\gamma)\right) \cup_{\varphi}\left(S^{1} \times E(K)\right)
$$

where the gluing map $\varphi$ is the diffeomorphism determined by $\varphi_{*}(\alpha)=m \mu_{K}+S^{1}$, $\varphi_{*}\left(\gamma^{\prime}\right)=\mu_{K}$, and $\varphi_{*}\left(\partial D^{2}\right)=\lambda_{K}$.

Definition 2.3 Suppose that $T \cong \alpha \times \gamma$ is the smooth torus in $X$ as above. Define

$$
\left(X, \Sigma_{K}(m)\right)=(X-N(T), \Sigma) \cup_{\varphi}\left(E(K) \times S^{1}, \varnothing\right) .
$$

This description means that performing a surgery on a smooth torus $T$ in $X$, we obtain $X$ again but $\Sigma$ might be changed. Now we need to check those two descriptions are the same definitions for our construction.

Lemma 2.4 Definition 2.1 and Definition 2.3 are equivalent.

Proof Given a knot $K$, recall that knotting the arc $I=I \times\{0\} \subset B^{3}=I \times B^{2}$ can be achieved by a cut-paste operation on the complement. Let $\gamma$ be an unknot which is the meridian of the arc $I$ in $B^{3}, E(K)$ be the exterior of the knot $K$ in $S^{3}$ and $N(\gamma)$ be the tubular neighborhood of $\gamma$ in $B^{3}$. If we replace the tubular neighborhood $N(\gamma)$ by $E(K)$ then we get $B^{3}$ with the knotted arc $K_{+}$instead of the trivial arc $I$. More precisely, note that $\left(B^{3}, K_{+}\right)=\left(v\left(\partial B^{3} \cup K_{+}\right), K_{+}\right) \cup E(K)$ where $v\left(\partial B^{3} \cup K_{+}\right)$ is the normal bundle in $B^{3}$ (see Figure 1). Let $\gamma^{\prime}$ be the push off of $\gamma$ onto $\partial N(\gamma)$.

Then there is a diffeomorphism $\left(B^{3}-N(\gamma), I\right) \rightarrow\left(\nu\left(\partial B^{3} \cup K_{+}\right), K_{+}\right)$mapping $\gamma^{\prime}$ to $\mu_{K}$ which induces a diffeomorphism

$$
h:\left(B^{3}-N(\gamma), I\right) \cup_{f} E(K) \longrightarrow\left(\nu\left(\partial B^{3} \cup K_{+}\right), K_{+}\right) \cup E(K)=\left(B^{3}, K_{+}\right),
$$

where $f: \partial N(\gamma) \longrightarrow \partial E(K)$ is a diffeomorphism determined by identifying $\gamma^{\prime}$ to $\mu_{K}$. Note that the diffeomorphism $h$ has $h(I)=K_{+}$and $\left.h\right|_{E(K)}=\mathrm{id}$.

Recalling the map $\tau$ defined in (1), we note that $h$ is the identity on $E(K)$ but $\tau$ is not, whereas on the outside of $E(K), \tau$ is the identity but $h$ is not. This implies 


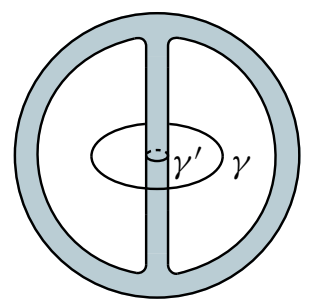

$\left(B^{3}, I\right)-N(\gamma)$

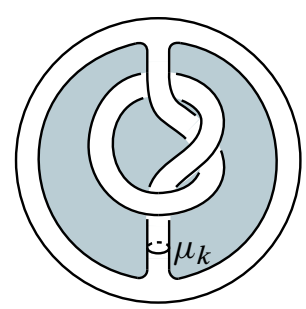

$\cup_{f} \quad E(K)$

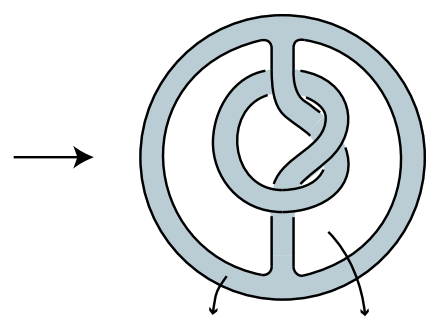

$v\left(\partial B^{3} \cup K_{-}\right) \cup E(K)$

Figure 1: Diffeomorphism $h:\left(B^{3}-N(\gamma), I\right) \cup_{f} E(K) \rightarrow\left(B^{3}, K_{+}\right)$

that $\tau$ is equivariant with respect to $h, \tau \circ h=h \circ \tau$. This $\tau$ induces a well-defined diffeomorphism mapping $[x, t]$ to $[h(x), t]$

$$
\left(\left(\left(B^{3}, I\right)-N(\gamma)\right) \cup_{f} E(K)\right) \times{ }_{\tau^{m}} S^{1} \longrightarrow\left(B^{3}, K_{+}\right) \times{ }_{\tau^{m}} S^{1} .
$$

Since $\tau^{m}$ is the identity on $\left(B^{3}, I\right)-N(\gamma),\left(\left(\left(B^{3}, I\right)-N(\gamma)\right) \cup_{f} E(K)\right) \times{ }_{\tau^{m}} S^{1}$ is the same as $\left(\left(B^{3}, I\right)-N(\gamma)\right) \times S^{1} \cup_{f \times 1} S^{1}\left(E(K) \times{ }_{\tau^{m}} S^{1}\right)$ and thus we have

$$
\left(\left(B^{3}, I\right)-N(\gamma)\right) \times S^{1} \cup_{f \times 1_{S^{1}}}\left(E(K) \times{ }_{\tau^{m}} S^{1}\right) \longrightarrow\left(B^{3}, K_{+}\right) \times_{\tau^{m}} S^{1} .
$$

Extending by the identity gives a diffeomorphism

$$
\begin{array}{r}
\left((X, \Sigma)-\left(B^{3}, I\right) \times S^{1}\right) \cup_{\partial}\left(\left(B^{3}, I\right)-N(\gamma)\right) \times S^{1} \cup_{f \times 1} S^{1}\left(E(K) \times_{\tau^{m}} S^{1}\right) \longrightarrow \\
\left((X, \Sigma)-\left(B^{3}, I\right) \times S^{1}\right) \cup_{\partial}\left(B^{3}, K_{+}\right) \times_{\tau^{m}} S^{1} .
\end{array}
$$

Rewriting

$$
\begin{aligned}
\left((X, \Sigma)-\left(B^{3}, I\right)\right. & \left.\times S^{1}\right) \cup_{\partial}\left(\left(B^{3}, I\right)-N(\gamma)\right) \times S^{1} \cup_{f \times 1_{S^{1}}}\left(E(K) \times{ }_{\tau^{m}} S^{1}\right) \\
& =X-N(\gamma) \times S^{1} \cup_{f \times 1_{S^{1}}}\left(E(K) \times{ }_{\tau^{m}} S^{1}\right) \\
& =X-\gamma \times D^{2} \times S^{1} \cup_{f \times 1_{S^{1}}}\left(E(K) \times{ }_{\tau^{m}} S^{1}\right),
\end{aligned}
$$

we get a diffeomorphism

$$
X-\gamma \times D^{2} \times S^{1} \cup_{f \times 1 S^{1}}\left(E(K) \times{ }_{\tau^{m}} S^{1}\right) \rightarrow\left((X, \Sigma)-\left(B^{3}, I\right) \times S^{1}\right) \cup_{\partial}\left(B^{3}, K_{+}\right) \times{ }_{\tau^{m}} S^{1} .
$$

Note that here the gluing map $f \times 1_{S^{1}}$ sends $\alpha$ to $S^{1}, \gamma^{\prime}$ to $\mu_{K}$ and $\partial D^{2}$ to $\lambda_{K}$ where $\mu_{K}$ and $\lambda_{K}$ are the meridian and the longitude of the knot $K$. Since $\tau^{m}$ is isotopic to identity, the isotopy induces a diffeomorphism $E(K) \times S^{1} \longrightarrow E(K) \times{ }_{\tau^{m}} S^{1}$. Again 
extending by the identity gives a diffeomorphism

$$
X-\gamma \times D^{2} \times S^{1} \cup_{f \times 1_{S^{1}}}\left(E(K) \times_{\tau^{m}} S^{1}\right) \rightarrow\left(X-\gamma \times D^{2} \times S^{1}\right) \cup_{\varphi}\left(E(K) \times S^{1}\right),
$$

where $\varphi$ is given by

$$
\begin{aligned}
\alpha & \longleftrightarrow S^{1}+m \mu_{K} \\
\gamma^{\prime} & \longleftrightarrow \mu_{K} \\
\partial D^{2} & \longleftrightarrow \lambda_{K} .
\end{aligned}
$$

Therefore the result follows.

\section{Diffeomorphism types}

Now let $X$ be a smooth simply connected $4-$ manifold and $\Sigma$ an embedded genus $g$ surface with self-intersection $n \geq 0$ and homology class $[\Sigma]=d \cdot \beta$, where $\beta$ is a primitive element in $H_{2}(X)$ and $\pi_{1}(X-\Sigma)=\mathbb{Z} / d$. Since $\Sigma$ is diffeomorphic to $T^{2} \# \ldots \# T^{2}$, let's choose a curve $\alpha$ whose image is the curve $\{p t\} \times S^{1}$ in the first $T^{2}=S^{1} \times S^{1}$. As we discussed in the previous section, a neighborhood of $\alpha$ in $X$ is of the form $\alpha \times I \times D^{2}=\alpha \times B^{3}$, where $\alpha \times I$ is in $\Sigma$. But we need to choose a certain trivialization of the normal bundle $v(\alpha \times I)$ in $X$ which will be used in Section 4 when we compute some topological invariants to identify the homeomorphism type of $\Sigma_{K}(m)$. It is possible to choose a trivialization $\sigma$ of $v(\alpha \times I)$ with the property that for some point $p \in \partial D^{2}, \sigma \mid \alpha \times\{0\} \times p$ is trivial in $H_{1}(X-\Sigma)$; we arbitrarily choose one trivialization $\sigma: \alpha \times I \times D^{2} \longrightarrow v(\alpha \times I)$ and let $\alpha^{\prime}$ be $\sigma \mid \alpha \times\{0\} \times p$ for some $p \in \partial D^{2}$. By composing $\sigma$ with a self diffeomorphism of $\alpha \times I \times D^{2}$ sending the element $\left(e^{i \theta}, t, z\right)$ to $\left(e^{i \theta}, t, e^{i k \theta} z\right)$ for an appropriate integer $k$, we can arrange $\alpha^{\prime}$ to be the zero homology element in $H_{1}(X-\Sigma) \cong \mathbb{Z} / d$, that is generated by the meridian $\sigma\left(p t \times \partial D^{2}\right)$ of $\Sigma$.

For a given $d$, the relation between $\Sigma_{K}(m)$ and $\Sigma$ depends somewhat on $m$. For example, if $d \not \equiv \pm 1(\bmod m)$ then for a nontrivial knot $K$, the surface $\Sigma_{K}(m)$ can be distinguished (even up to homeomorphism) from $\Sigma$ by considering the fundamental group $\pi_{1}\left(X-\Sigma_{K}(m)\right)$. First, we need to understand the explicit expression of this group.

In this paper, we will denote by $(X, Y)^{d}$ a $d$-fold covering of $X$ branched along $Y$.

Lemma 3.1 Let $\mu$ be the meridian of the knotted arc $K_{+}$and let the base point $*$ be in $\partial E(K)=K \times \partial D^{2} \times\{0\}$. Then

$\pi_{1}\left(X-\Sigma_{K}(m)\right)=\left\langle\pi_{1}\left(B^{3}-K_{+}, *\right)\right| \mu^{d}=1, \beta=\tau_{*}^{m}(\beta)$, for all $\left.\beta \in \pi_{1}\left(B^{3}-K_{+}, *\right)\right\rangle$. 
Proof Considering the definition of $\left(X, \Sigma_{K}(m)\right)$, we have that the complement of $\Sigma_{K}(m)$ in $X, X-\Sigma_{K}(m)$, is $\left(X-S^{1} \times B^{3}-\Sigma\right) \cup S^{1} \times{ }_{\tau^{m}}\left(B^{3}-K_{+}\right)$. Then we get that the intersection of the two components in the decomposition is

$$
\left(X-S^{1} \times B^{3}-\Sigma\right) \cap S^{1} \times_{\tau^{m}}\left(B^{3}-K_{+}\right)=S^{1} \times\left(\partial B^{3}-\{\text { two points }\}\right) .
$$

Here we need to note that the action of $\tau$ on $\partial B^{3}-\{$ two points $\}$ is trivial. Then using Van Kampen's theorem for this decomposition, we have the following diagram:

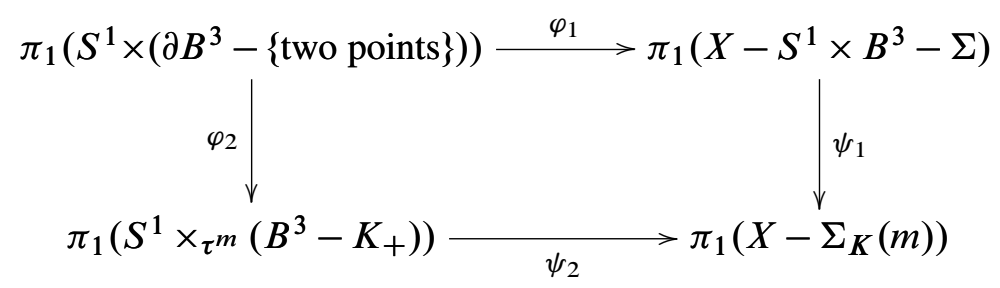

Note that $X-S^{1} \times B^{3}-\Sigma$ is homotopy equivalent to $X-\Sigma$ and $\pi_{1}(X-\Sigma) \cong \mathbb{Z} / d$ is generated by the meridian $\gamma$ of $\Sigma$. We also know that $\pi_{1}\left(S^{1} \times\left(\partial B^{3}-\{\right.\right.$ two points $\left.\left.\}\right)\right)$ is generated by $\left[S^{1}\right]$, which is identified with the class of the curve $\alpha^{\prime}$ pushed off along a given trivialization of neighborhood of $\alpha$, and by $\mu$. Since the meridian $\mu$ of the knot is identified with $\gamma, \varphi_{1}$ is onto and so $\psi_{2}$ is also onto. Moreover, $\operatorname{ker} \psi_{2}=\left\langle\varphi_{2}\left(\operatorname{ker} \varphi_{1}\right)\right\rangle$. Since $\operatorname{ker} \varphi_{1}=\left\langle\alpha^{\prime}, \mu^{d}\right\rangle$ and

$$
\begin{aligned}
& \pi_{1}\left(S^{1} \times_{\tau^{m}}\left(B^{3}, K_{+}\right)\right)= \\
& \left.\qquad \pi_{1}\left(B^{3}-K_{+}\right), \alpha^{\prime} \mid \alpha^{\prime-1} \beta \alpha^{\prime}=\tau_{*}^{m}(\beta) \text { for all } \beta \in \pi_{1}\left(B^{3}-K_{+}\right)\right\rangle,
\end{aligned}
$$

it follows that

$$
\begin{aligned}
& \pi_{1}\left(X-\Sigma_{K}(m)\right) \\
& \left.=\left\langle\pi_{1}\left(B^{3}-K_{+}\right), \alpha^{\prime}\right| \alpha^{\prime}=1, \mu^{d}=1, \alpha^{\prime-1} \beta \alpha^{\prime}=\tau_{*}^{m}(\beta) \text { for all } \beta \in \pi_{1}\left(B^{3}-K_{+}\right)\right\rangle \\
& \left.=\left\langle\pi_{1}\left(B^{3}-K_{+}\right)\right| \mu^{d}=1, \beta=\tau_{*}^{m}(\beta) \text { for all } \beta \in \pi_{1}\left(B^{3}-K_{+}\right)\right\rangle .
\end{aligned}
$$

which completes the proof.

The following example shows that we can distinguish $\Sigma_{K}(m)$ using $\pi_{1}$.

Example 3.2 For any nontrivial knot $K$, let $d=2$, ie $\pi_{1}(X-\Sigma)=\mathbb{Z} / 2$, and let $m$ be any even number. If we consider the fundamental group $\pi_{1}\left(X-\Sigma_{K}(m)\right)$, then by Lemma 3.1,

$\pi_{1}\left(X-\Sigma_{K}(m)\right)=\left\langle\pi_{1}\left(B^{3}-K_{+}, *\right)\right| \mu^{d}=1, \beta=\tau_{*}^{m}(\beta)$ for all $\left.\beta \in \pi_{1}\left(B^{3}-K_{+}, *\right)\right\rangle$, 
where $\mu$ is the meridian of the knotted arc $K_{+}$and the base point $*$ is in $\partial E(K)=$ $K \times \partial D^{2} \times\{0\}$.

Recall the group of the knot $\pi_{1}\left(B^{3}-K_{+}, *\right)$ has the Wirtinger presentation

$$
\left\langle g_{1}, g_{2}, \ldots, g_{n} \mid r_{1}, r_{2}, \ldots, r_{n}\right\rangle
$$

where $g_{1}=\mu$ and other generators $g_{i}$ represent the loop that, starting from a base point, goes straight to the $i^{\text {th }}$ over-passing arc in the knot diagram, encircles it and returns to the base point.

Note that $\tau_{*}^{m}\left(g_{1}\right)=g_{1}$ and $\tau_{*}^{m}\left(g_{i}\right)=g_{1}^{-m} g_{i} g_{1}^{m}$ for other generators $g_{i}$ by the definition of $\tau$. Since $d=2$ ie $g_{1}^{2}=1$ and $m$ is an even number, $\tau_{*}^{m}\left(g_{i}\right)=g_{1}^{-m} g_{i} g_{1}^{m}$ is always $g_{i}$ and thus we get

$$
\pi_{1}\left(X-\Sigma_{K}(m)\right)=\pi_{1}\left(B^{3}-K_{+}\right) / \mu^{2}=\pi_{1}\left(S^{3}-K\right) / \mu^{2} .
$$

If we take a 2-fold branched cover $\left(S^{3}, K\right)^{2}$ along the knot $K$ then the fundamental group $\pi_{1}\left(\left(S^{3}, K\right)^{2}\right)$ is same as the group $\pi_{1}\left(\left(S^{3}-K\right)^{2}\right) / \tilde{\mu}$, where $\left(S^{3}-K\right)^{2}$ is the 2-fold unbranched cover and $\tilde{\mu}$ is a lift of $\mu$. So $\pi_{1}\left(S^{3}-K\right) / \mu^{2}$ has $\pi_{1}\left(\left(S^{3}, K\right)^{2}\right)$ as an index 2 subgroup. The Smith conjecture [12] states that for any $d \geq 1$, the fundamental group of a $d$-fold branched cover $\pi_{1}\left(\left(S^{3}, K\right)^{d}\right)$ is nontrivial unless $K$ is a trivial knot. Hence $\pi_{1}\left(X-\Sigma_{K}(m)\right)$ has a nontrivial index 2 subgroup and so

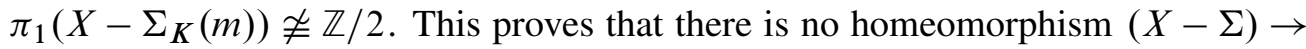
$\left(X-\Sigma_{K}(m)\right)$.

A more interesting case is when $\pi_{1}$ does not distinguish the embedding of $\Sigma_{K}(m)$, so that we have to use other means to show that $\Sigma$ is not diffeomorphic to $\Sigma_{K}(m)$. In particular, for the case $d \equiv \pm 1(\bmod m)$, we have:

Proposition 3.3 If $d \equiv \pm 1(\bmod m)$ then $\pi_{1}(X-\Sigma)=\pi_{1}\left(X-\Sigma_{K}(m)\right)=\mathbb{Z} / d$.

Proof If $d=1$ then by Lemma 3.1, $\pi_{1}(X-\Sigma)=\pi_{1}\left(X-\Sigma_{K}(m)\right)=\{1\}$. So, we assume $d>1$. To express $\pi_{1}(X-\Sigma)$ more explicitly, in a Wirtinger presentation of the knot group $\pi_{1}\left(B^{3}-K_{+}, *\right)$, choose meridians $g_{j}$ conjugate to the meridian $g_{1}=\mu$ of the knot $K$ for each $j=2, \ldots, n$ as generators of $\pi_{1}\left(B^{3}-K_{+}\right)$. Then with Lemma 3.1, we represent $\pi_{1}\left(X-\Sigma_{K}(m)\right)$ by

$$
\left.\left\langle g_{1}, g_{2}, \ldots, g_{n}\right| g_{1}^{d}=1, r_{1}, \ldots, r_{n}, \beta=\tau_{*}^{m}(\beta) \text { for all } \beta \in \pi_{1}\left(B^{3}-K_{+}\right)\right\rangle
$$

where $r_{1}, \ldots, r_{n}$ are relations of $\pi_{1}\left(B^{3}-K_{+}\right)$. 
Considering the definition of $\tau, \tau_{*}(\mu)=\mu$ and $\tau_{*}\left(g_{j}\right)=\mu^{-1} g_{j} \mu$ for each $j=$ $2, \ldots, n$ so that we rewrite

$$
\begin{aligned}
& \pi_{1}\left(X-\Sigma_{K}(m)\right) \\
& \left.\quad=\left\langle g_{1}, g_{2}, \ldots, g_{n}\right| g_{1}^{d}=1, r_{1}, \ldots, r_{n}, g_{j}=g_{1}^{-m} g_{j} g_{1}^{m} \text { for } j=2, \ldots, n\right\rangle .
\end{aligned}
$$

Now we claim that this is equal to $\left\langle g_{1}, g_{2}, \ldots, g_{n}\right| g_{1}^{d}, r_{1}, \ldots, r_{n}, g_{1}=g_{1}^{-1} g_{j} g_{1}$ for $j=2, \ldots, n\rangle$.

Since $d \equiv \pm 1(\bmod m)$, we can write $d=m k \pm 1$ for some integer $k$. Let $l=d-m$. Then $l=d-m=m k \pm 1-m=m(k-1) \pm 1$.

$$
\begin{array}{rlr}
g_{j}=g_{1}^{-m} g_{j} g_{1}^{m} & \Longrightarrow g_{1}^{-l} g_{j} g_{1}^{l}=g_{1}^{-l}\left(g_{1}^{-m} g_{j} g_{1}^{m}\right) g_{1}^{l} \\
& \Longrightarrow g_{1}^{-l} g_{j} g_{1}^{l}=g_{1}^{-(l+m)} g_{j} g_{1}^{(l+m)}=g_{j} & (l+m=d) \\
& \Longrightarrow g_{1}^{-m(k-1) \mp 1} g_{j} g_{1}^{m(k-1) \pm 1}=g_{j} \\
& \Longrightarrow g_{1}^{\mp 1}\left(g_{1}^{-m(k-1)} g_{j} g_{1}^{m(k-1)}\right) g_{1}^{ \pm 1}=g_{j} \ldots
\end{array} \quad(l=m(k-1) \pm 1)
$$

We claim that $g_{1}^{-m(k-1)} g_{j} g_{1}^{m(k-1)}=g_{j}$; if $k-1=0$ or 1 then it is clearly true. Let's assume that it is true for $k-1=i$. For $k-1=i+1$, by induction

$$
g_{1}^{-m(i+1)} g_{j} g_{1}^{m(i+1)}=g_{1}^{-m i}\left(g_{1}^{-m} g_{j} g_{1}^{m}\right) g_{1}^{m i}=g_{1}^{-m i} g_{j} g_{1}^{m i}=g_{j} .
$$

This implies that $(*)$ becomes $g_{1}^{\mp 1} g_{j} g_{1}^{ \pm 1}=g_{j}$ and so we now get

$$
\begin{aligned}
& \pi_{1}\left(X-\Sigma_{K}(m)\right) \\
& \left.\quad=\left\langle g_{1}, g_{2}, \ldots, g_{n}\right| g_{1}^{d}=1, r_{1}, \ldots, r_{n},\left[g_{1}, g_{j}\right]=1 \text { for } j=2, \ldots, n\right\rangle .
\end{aligned}
$$

If we consider the Wirtinger presentation of the knot group then we can show $g_{1}=$ $g_{2}=\ldots=g_{n}$ with the relations $r_{1}, . ., r_{n}$ and $\left[g_{1}, g_{j}\right]$; corresponding to the following crossing, the relator gives $g_{2} g_{s}=g_{s} g_{1}$ or $g_{s} g_{2}=g_{1} g_{s}$.
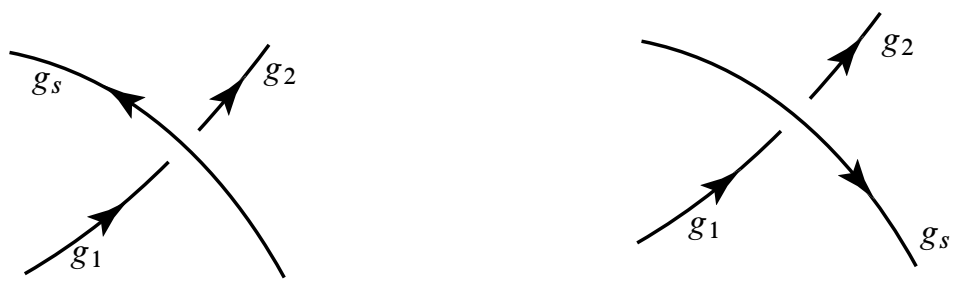

Figure 2: Wirtinger presentation of the knot group 
So, $g_{1}=g_{2}$. By an induction argument, we can conclude that $g_{1}=g_{2}=\ldots=g_{n}$. This proves that

$$
\pi_{1}\left(X-\Sigma_{K}(m)\right)=\left\langle\mu \mid \mu^{d}=1\right\rangle \cong \mathbb{Z} / d .
$$

Remark The same technique works for many other cases, for example if $d=2$ and $m$ is an odd integer.

We can also distinguish some $\Sigma_{K}(m)$ smoothly by using relative Seiberg-Witten (SW) theory, following the technique of Fintushel and Stern [2]. In [4], they introduced a method called 'knot surgery' modifying a 4-manifold while preserving its homotopy type by using a knot in $S^{3}$ and also gave a formula for the SW-invariant of the new manifold to detect the diffeomorphism type under suitable circumstances.

Let $X$ be a smooth 4-manifold and $T$ in $X$ be an imbedded 2-torus with trivial normal bundle. (In [14], C Taubes showed the ' $c$-embedded' condition on the torus in the original paper [4] to be unnecessary.) Then the knot surgery may be described as follows.

Let $K$ be a knot in $S^{3}$, and $K \times D^{2}$ be the trivialization of its open tubular neighborhood given by the 0 -framing. Let $\varphi: \partial\left(T \times D^{2}\right) \longrightarrow \partial\left(K \times D^{2}\right) \times S^{1}$ be any diffeomorphism with $\varphi\left(p \times \partial D^{2}\right)=K \times q$ where $p \in T, q \in \partial D^{2} \times S^{1}$ are points. Define

$$
X_{K}=\left(X-T \times D^{2}\right) \cup_{\varphi} E(K) \times S^{1} .
$$

In our situation, the surgical construction of $\Sigma_{K}(m)$ is performing a surgery on a torus $T$ in $X$ called a 'rim torus'. Recall the torus $T$ has the form $\gamma \times \alpha$ where $\gamma$ is the meridian of $\Sigma$ and $\alpha$ is a curve in $\Sigma$ (see Lemma 2.4). In other words, we remove a neighborhood of the torus and sew in $E(K) \times S^{1}$ along the gluing map given in Definition 2.3. Considering this identification, we can observe that the pair $\left(X, \Sigma_{K}(m)\right)$ is obtained by a knot surgery.

Fintushel and Stern wrote a note to fill a gap in the proof of the main theorem in [3]. In the note [2], they explained the effect of rim surgery on the relative SeibergWitten invariant of $X-\Sigma$. The $m$-twist rim surgery on $X-\Sigma$ affects its relative Seiberg-Witten invariant exactly same as rim surgery. So we will refer to the note [2] to distinguish the pairs $(X, \Sigma)$ and $\left(X, \Sigma_{K}(m)\right)$ smoothly.

If the self-intersection $\Sigma \cdot \Sigma=n \geq 0$, blow up $X n$ times to get a pair $\left(X_{n}, \Sigma_{n}\right)$ and reduce the self intersection to zero. For simplicity, we may assume that $\Sigma \cdot \Sigma=0$. In general, the relative Seiberg-Witten invariant $S W_{X, \Sigma}$ is an element in the Floer homology of the boundary $\Sigma \times S^{1}$ [10]. We restrict $S W_{X, \Sigma}$ to the set $\mathcal{T}$ which is the 
collection of $\operatorname{spin}^{c}$-structures $\tau$ on $X-N(\Sigma)$ whose restriction to $\partial N(\Sigma)$ is the $\operatorname{spin}^{c}-$ structure $\pm s_{g-1}$ corresponding to the element $(g-1,0)$ of $H^{2}\left(\Sigma \times S^{1}\right) \cong \mathbb{Z} \oplus H^{1}(\Sigma)$. Then we obtain a well-defined integer-valued Seiberg-Witten invariant $S W_{X, \Sigma}^{\mathcal{T}}$ and so get a Laurent polynomial $S W_{X, \Sigma}^{\mathcal{T}}$ with variables in

$$
A=\left\{\alpha \in H^{2}(X-\Sigma)|\alpha|_{\Sigma \times S^{1}}= \pm s_{g-1}\right\} .
$$

If there is a diffeomorphism $f:(X, \Sigma) \rightarrow\left(X^{\prime}, \Sigma^{\prime}\right)$ then it induces a map $f^{*}: A^{\prime} \rightarrow A$ sending $S W_{X^{\prime}, \Sigma^{\prime}}^{\mathcal{T}}$ to $S W_{X, \Sigma}^{\mathcal{T}}$.

Theorem 3.4 Suppose the relative Seiberg-Witten invariant $S W_{X, \Sigma}^{\mathcal{T}}$ is nontrivial. If there is a diffeomorphism $\left(X, \Sigma_{K}(m)\right) \longrightarrow\left(X, \Sigma_{J}(m)\right)$ then the set of coefficients (with multiplicity) of $\Delta_{K}(t)$ is equal to that of $\Delta_{J}(t)$, where $\Delta_{K}(t)$ and $\Delta_{J}(t)$ are the Alexander polynomials of $K$ and $J$ respectively.

Proof If there is a pairwise diffeomorphism $\left(X, \Sigma_{K}(m)\right) \longrightarrow\left(X, \Sigma_{J}(m)\right)$ then it induces a diffeomorphism $\left(X_{n}, \Sigma_{n, K}(m)\right) \longrightarrow\left(X_{n}, \Sigma_{n, J}(m)\right)$. So, we now may assume that $\Sigma \cdot \Sigma=0$.

According to the note [2], the proof of the knot surgery theorem [4] works in the relative case to show that

$$
S W_{(X-\Sigma)_{K}}^{\mathcal{T}}=S W_{X, \Sigma}^{\mathcal{T}} \cdot \Delta_{K}\left(r^{2}\right)
$$

where $r=[T]$ is the element of $R$, the subgroup of $H^{2}(X-\Sigma)$ generated by the rim torus $T$ of $\Sigma$. Note that the rim torus $T$ is homologically essential in $X-\Sigma$.

Since the relative Seiberg-Witten invariant $S W_{X, \Sigma_{K}(m)}^{\mathcal{T}}=S W_{(X-\Sigma)_{K}}^{\mathcal{T}}$, applying the knot surgery theorem to the $m$-twist rim surgery we also get that the coefficients of $S W_{X, \Sigma}^{\mathcal{T}} \cdot \Delta_{K}\left(r^{2}\right)$ must be equal to those of $S W_{X, \Sigma}^{\mathcal{T}} \cdot \Delta_{J}\left(r^{\prime 2}\right)$.

Remark (1) The theorem implies that for $\Delta_{K}(t) \neq 1,(X, \Sigma)$ is not pairwise diffeomorphic to $\left(X, \Sigma_{K}(m)\right)$.

(2) In [3] standard pairs $\left(Y_{g}, S_{g}\right)$ were defined where $Y_{g}$ is a simply connected Kähler surface, $S_{g}$ is a primitively embedded genus $g \geq 1$ Riemann surface in $Y_{g}$ with $S_{g} \cdot S_{g}=0$. According to the note [2], the hypothesis $S W_{X \#_{\Sigma}=S_{g}} Y_{g} \neq 1$ of [3] implies $S W_{X, \Sigma}^{\mathcal{T}} \neq 1$ by the gluing formula [10].

(3) $S W_{X \#_{\Sigma=S g} Y_{g}}$ is nontrivial when $\Sigma$ is a complex curve in a complex surface.

The case of curves in $\mathbb{C} \mathbf{P}^{2}$ is particularly interesting. By applying Theorem 3.4, we obtain the following corollary. 
Corollary 3.5 For $d>2$ with $d \equiv \pm 1(\bmod m)$, if $\Sigma$ is a degree $d$-curve in $\mathbb{C} \mathbf{P}^{2}$ then $\left(\mathbb{C} \mathbf{P}^{2}, \Sigma\right)$ is not pairwise diffeomorphic to $\left(\mathbb{C} \mathbf{P}^{2}, \Sigma_{K}(m)\right)$ for any knot $K$ with $\Delta_{K}(t) \neq 1$, but $\pi_{1}\left(\mathbb{C} \mathbf{P}^{2}-\Sigma_{K}(m)\right) \cong \mathbb{Z} / d$.

Proof Note that $\Sigma$ is a symplectically embedded surface with positive genus $g=$ $\frac{1}{2}(d-1)(d-2)$. Under the construction in [3], $S_{g}$ is also symplectically embedded in $Y_{g}$ since $S_{g}$ is a complex submanifold of the Kähler manifold $Y_{g}$. Since the group $\pi_{1}\left(C \mathbf{P}^{2}-\Sigma\right)=\mathbb{Z} / d$, note that $\pi_{1}\left(C \mathbf{P}^{2}-\Sigma_{K}(m)\right)=\mathbb{Z} / d$ by Proposition 3.3.

Let us denote by $C P_{d^{2}}^{2}$ the manifold obtained by blowing up $d^{2}$ times $C P^{2}$. Then $C P_{d^{2}}^{2} \#_{\Sigma_{d^{2}}}=S_{g} Y_{g}$ is also a symplectic manifold by Gompf [7]. So (see Taubes [13]),

$$
S W_{C P_{d^{2}}^{2} \#_{\Sigma_{d^{2}}}=S_{g} Y_{g}} \neq 0 .
$$

By Theorem 3.4, the result follows.

This means that for any $d \geq 3$, there are infinitely many smooth oriented closed surfaces $\Sigma$ in $\mathbb{C} \mathbf{P}^{2}$ representing the class $d h \in H_{2}\left(\mathbb{C} \mathbf{P}^{2}\right)$, where $h$ is a generator of $H_{2}\left(\mathbb{C} \mathbf{P}^{2}\right)$, having genus $(\Sigma)=\frac{1}{2}(d-1)(d-2)$ and $\pi_{1}\left(\mathbb{C} \mathbf{P}^{2}-\Sigma\right) \cong \mathbb{Z} / d$, such that the pairs $\left(\mathbb{C} \mathbf{P}^{2}, \Sigma\right)$ are pairwise smoothly non-equivalent. Such examples, for $d \geq 5$, were known by the work of Finashin which we describe in order to contrast it with our construction. In [1], he constructed a new surface by knotting a standard one along a suitable annulus membrane.

More precisely, let $X$ be a 4-manifold and $\Sigma$ be a smoothly embedded surface. Suppose that there is a smoothly embedded surface $M$ in $X$, called a 'membrane', such that $M \cong S^{1} \times I, M \cap \Sigma=\partial M$ and $M$ meets to $\Sigma$ normally along $\partial M$. By adjusting a trivialization of its regular neighborhood $U$, we can assume that $U\left(\cong S^{1} \times D^{3}\right) \cap \Sigma=$ $S^{1} \times f$, where $f=I_{0} \sqcup I_{1}=I \times \partial I$ is a disjoint union of two unknotted segments of a part of the boundary of a band $b=I \times I$ in $D^{3}$. Here the band $b=I \times I$ is trivially embedded in $D^{3}$ and the intersection $I \times I \cap \partial D^{3}=\partial I \times I$ (see Figure 3).

Then given a knot $K$ in $S^{3}$, we can get a new surface $\Sigma_{K, F}$ by knotting $f$ along $K$ in $D^{3}$ (see Figure 4).

In [1], Finashin showed that we can find such a membrane $M$ in $\mathbb{C} \mathbf{P}^{2}$ and proved that $\left(\mathbb{C} \mathbf{P}^{2}, \Sigma_{K, F}\right)$ is pairwise non-equivalent to $\left(\mathbb{C} \mathbf{P}^{2}, \Sigma\right)$ for an algebraic curve $\Sigma$ of degree $d \geq 5$. In particular, for an even degree he showed that the double cover branched along $\Sigma_{K, F}$ is diffeomorphic to the 4-manifold obtained from the double cover branched along $\Sigma$ by knot surgery along the torus, which is the pre-image of the membrane $M$ in the covering, via the knot $K \# K$. So, the knot surgery theorem in [4] distinguishes the branch covers by comparing their SW-invariants. For odd cases, one 


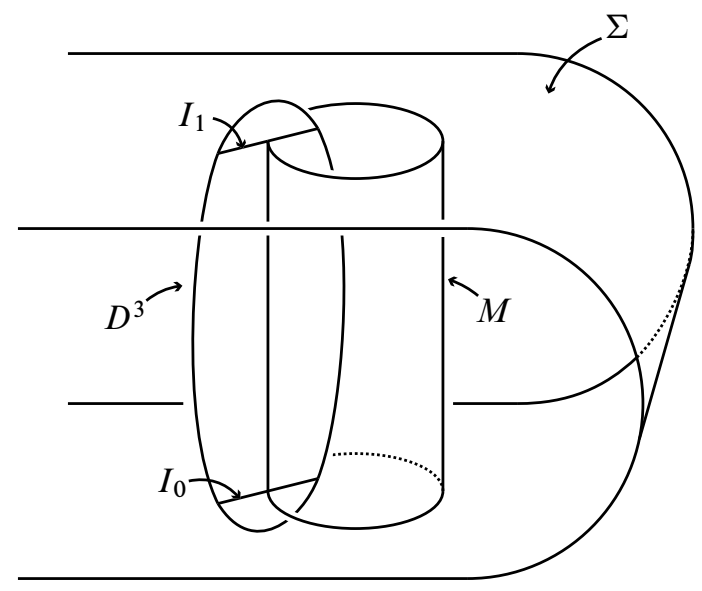

Figure 3: $\left(\mathbb{C} \mathbf{P}^{2}, \Sigma\right)$
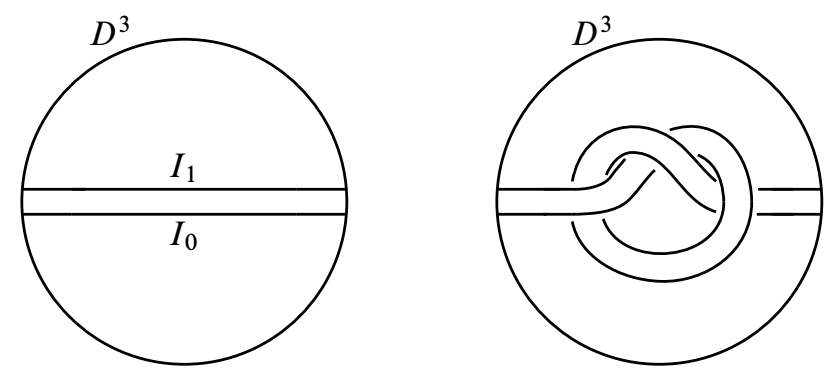

Figure 4: $\left(D^{3}, I \times I\right)$ and $\left(D^{3}, K_{+} \times I\right)$

can use the same argument using $d$-fold coverings to show smooth non-equivalence of embeddings.

Our examples constructed by twist spinning are different from Finashin's for a degree $d \geq 5$. To see this, we compute the SW-invariant of the branched cover of $\left(\mathbb{C} \mathbf{P}^{2}, \Sigma_{K}(m)\right)$. Let $Y$ be a $d$-fold branch cover along $\Sigma$ and $Y_{K, m}$ be a $d$-fold branch cover along $\Sigma_{K}(m)$. Let's consider the description for the branch cover $Y_{K, m}$. We write $Y_{K, m}$ as the union of two $d$-fold branched covers:

$$
\left(Y_{K, m}, \Sigma_{K}(m)\right)=\left(X-S^{1} \times B^{3}, \Sigma-S^{1} \times I\right)^{d} \cup_{\partial}\left(S^{1} \times_{\tau^{m}}\left(B^{3}, K_{+}\right)\right)^{d}
$$

Since the homology group $H_{1}\left(X-S^{1} \times B^{3}-\Sigma\right) \cong H_{1}(X-\Sigma) \cong \mathbb{Z} / d$, the branch cover $\left(X-S^{1} \times B^{3}, \Sigma-S^{1} \times I\right)^{d}$ is unique and is the same as $Y-S^{1} \times B^{3}$. We 
also need to note that $\left(S^{1} \times{ }_{\tau^{m}}\left(B^{3}, K_{+}\right)\right)^{d}=S^{1} \times \tilde{\tau}^{m}\left(B^{3}, K_{+}\right)^{d}$ for some lift $\tilde{\tau}^{m}$ of $\tau^{m}$ which is referred to in the proof for Proposition 4.3. So we rewrite

$$
\left(Y_{K, m}, \Sigma_{K}(m)\right)=\left((Y, \Sigma)-S^{1} \times\left(B^{3}, I\right)\right) \cup_{S^{1} \times S^{2}}\left(S^{1} \times_{\tilde{\tau}^{m}}\left(B^{3}, K_{+}\right)^{d}\right) .
$$

If $K$ is any knot with the homology $H_{1}\left(\left(S^{3}-K\right)^{d}\right) \cong \mathbb{Z}$ then $S^{1} \times \tilde{\tau}^{m}\left(B^{3}, K_{+}\right)^{d}$ is homologically equivalent to $S^{1} \times B^{3}$. We may look at knots, introduced in Section 4, having the property that their $d$-fold covers are homology circles. An extension of the result of Vidussi in [16] shows

$$
S W_{Y_{K, m}}=S W_{Y} .
$$

But the SW-invariant of branched cover along the surface $\Sigma_{K, F}$ constructed by Finashin is not standard as we saw above. Our examples also cover the case of degree $d=3$ and 4 which were not treated in his paper.

Remark By the same argument in Fintushel and Stern [3], we can also say that if $X$ is a simply connected symplectic 4 -manifold and $\Sigma$ is a symplectically embedded surface then $\Sigma_{K}(m)$ is not smoothly ambient isotopic to a symplectic submanifold of $X$ for $\Delta_{K}(t) \neq 1$. Using Taubes' result in [13], we can easily get a proof of this (see [3] for more detail).

\section{Homeomorphism types}

In this section, we shall investigate when $\Sigma_{K}(m)$ is topologically equivalent to $\Sigma$. As we saw in the previous section, in the case $d \equiv \pm 1(\bmod m)$ their complements in $X$ have the same fundamental group. So, for this case one would like to show that they are pairwise homeomorphic under a certain condition by constructing an explicit $s$-cobordism. Note that it is not known if Finashin's examples are topologically unknotted [1, Remark, p50]. Recall that the $s$-cobordism theorem gives a way for showing manifolds are homeomorphic.

Let $W$ be a compact $n$-manifold with the boundary being the disjoint union of manifolds $M_{0}$ and $M_{1}$. Then the original $s$-cobordism theorem states that for $n \geq 6$, $W$ is diffeomorphic to $M_{0} \times[0,1]$ exactly when the inclusions of $M_{0}$ and $M_{1}$ in $W$ are homotopy equivalences and the Whitehead torsion $\tau\left(W, M_{0}\right)$ in $\mathrm{Wh}\left(\pi_{1}(W)\right)$ is zero. By the work of M Freedman [6], the $s$-cobordism theorem is known to hold topologically in the case $n=5$ when $\pi_{1}(W)$ is poly-(finite or cyclic). A relative $s$-cobordism theorem also holds.

To make use of those theorems we shall construct a relative $h$-cobordism from $X-v(\Sigma)$ to $X-v\left(\Sigma_{K}(m)\right)$ and then apply the relative $s$-cobordism theorem. 
First consider the following situation. Let $K$ be a ribbon knot in $S^{3}$ so that $\left(S^{3}, K\right)=$ $\partial\left(B^{4}, \Delta\right)$ for some ribbon disc $\Delta$ in $B^{4}$. By Lemma 3.1 in [8], $\pi_{1}\left(S^{3}-K\right) \longrightarrow$ $\pi_{1}\left(B^{4}-\Delta\right)$ is surjective. Take out a 4 -ball $\left(B^{\prime}, B^{\prime} \cap \Delta\right)$ from the interior of $\left(B^{4}, \Delta\right)$ such that $B^{\prime} \cap \Delta$ is an unknotted disk (see Figure 5).

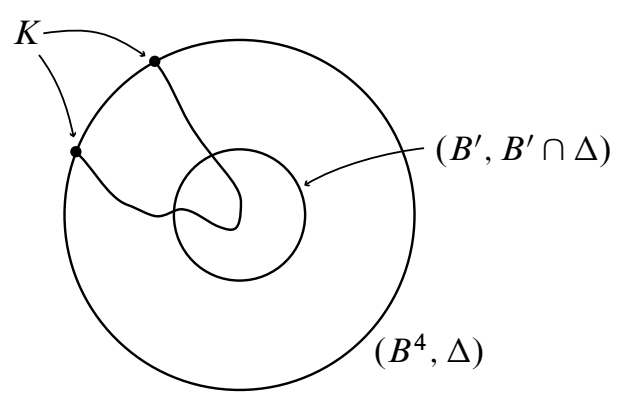

Figure 5: Ribbon disk in $B^{4}$

Let $A=\Delta-\left(B^{\prime} \cap \Delta\right)$ then we can easily note that $A$ is a concordance between $K$ and an unknot $O$. Let $K=K_{+} \cup K_{-}$where $K_{+}$is a knotted arc and $K_{-}$is a trivial arc diffeomorphic to $I$. Write $S^{3}=B_{+}^{3} \cup B_{-}^{3}$ where $B_{+}^{3}, B_{-}^{3}$ are 3-balls. Let's assume that $B_{-}^{3} \times I \subset S^{3} \times I$ with $\left(B_{-}^{3} \times I, B_{-}^{3} \times I \cap A\right)=\left(B_{-}^{3} \times I, I \times I\right)$ and $\left(B_{-}^{3} \times 1, B_{-}^{3} \times 1 \cap A\right)=\left(B_{-}^{3} \times 1, K_{-}\right)$.

If we take out $B_{-}^{3} \times I$ from $S^{3} \times I$ then we are left with $\left(S^{3} \times I, A\right)-\left(B_{-}^{3} \times I, I \times I\right)=$ $\left(B_{+}^{3} \times I, A-I \times I\right)$. Denoting $A-I \times I$ by $A_{+}$, we have $B_{+}^{3} \times 1 \cap A_{+}=K_{+}$and $B_{+}^{3} \times 0 \cap A_{+}=O_{+}$where $O_{+}$is a trivial arc of $O$ (see Figure 6).

We will define a self diffeomorphism on $\left(S^{3} \times I, A\right)$ in the same way that we defined the twist map in Section 2. Recall $\left(S^{3} \times I, A\right)-\left(B_{-}^{3} \times I, I \times I\right)=\left(B_{+}^{3} \times I, A_{+}\right)$. Note the normal bundle $v(A)$ in $S^{3} \times I$ is $A \times D^{2}$ and let $E(A)$ be the exterior $\operatorname{cl}\left(S^{3} \times I-A \times D^{2}\right)$ of $A$ in $S^{3} \times I$. Then $E(A)$ coincides (up to isotopy), with $\operatorname{cl}\left(B_{+}^{3} \times I-A_{+} \times D^{2}\right)$. Thus, $\partial E(A)=A \times \partial D^{2}$ is $\partial\left(\operatorname{cl}\left(B_{+}^{3} \times I-A_{+} \times D^{2}\right)\right) \cong T \times I$ where $T$ is a torus. Let $A \times \partial D^{2} \times I$ be the collar of $\partial E(A)$ in $E(A)$. Define $\tau:\left(S^{3} \times I, A\right) \longrightarrow\left(S^{3} \times I, A\right)$ by

$$
\tau\left(x \times e^{i \theta} \times t\right)=x \times e^{i(\theta+2 \pi t)} \times t \quad \text { for } \quad x \times e^{i \theta} \times t \in A \times \partial D^{2} \times I
$$

and $\tau(y)=y$ for $y \notin A \times \partial D^{2} \times I$.

Then note that $\tau$ is the identity on a neighborhood of $A_{+}$and that $\left.\tau\right|_{B_{+}^{3} \times 0-O_{+}}$and $\left.\tau\right|_{B_{+}^{3} \times 1-K_{+}}$are the twist maps induced by the unknot $O$ and the knot $K$ defined in 


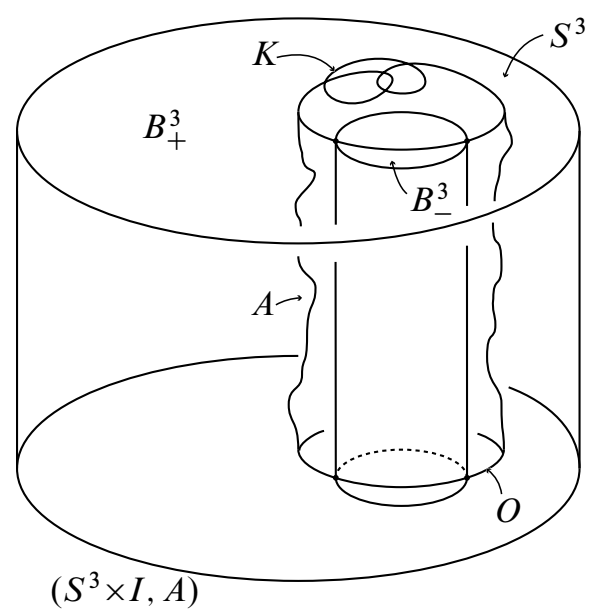

Figure 6: A concordance between $K$ and unknot

(1). Denote those maps by $\tau_{O}$ and $\tau_{K}$ respectively. Using this diffeomorphism $\tau$, we can also construct a new submanifold $(\Sigma \times I)_{A}(m)$ from an embedded manifold $\Sigma \times I$ to $X \times I$ in the way to construct a new surface $\Sigma_{K}(m)$.

Definition 4.1 Under the above notation, define

$$
\left(X \times I,(\Sigma \times I)_{A}(m)\right)=X \times I-S^{1} \times\left(B^{3} \times I, I \times I\right) \cup S^{1} \times \tau^{m}\left(B^{3} \times I, A_{+}\right) .
$$

Then we can easily note that

$$
\begin{aligned}
& X \times 1=X-S^{1} \times\left(B^{3} \times 1, I \times 1\right) \cup S^{1} \times{ }_{\tau_{K}^{m}}\left(B^{3} \times 1, K_{+}\right)=\left(X, \Sigma_{K}(m)\right), \\
& X \times 0=X-S^{1} \times\left(B^{3} \times 0, I \times 0\right) \cup S^{1} \times_{\tau_{O}^{m}}\left(B^{3} \times 0, O_{+}\right)=(X, \Sigma)
\end{aligned}
$$

and so the complement $X \times I-(\Sigma \times I)_{A}(m)$ gives a concordance between $X-\Sigma$ and $X-\Sigma_{K}(m)$ (See Figure 7). We will denote this concordance by $W$ and will later show this $W$ is a $h$-cobordism under certain conditions. Here we note that the cobordism $W$ is a product near the boundary. To see what conditions are needed, consider several other properties first.

Recall for any pair $(X, Y)$, we denote by $X^{d}$ a $d$-fold cover of $X$ and $(X, Y)^{d}$ a $d$-fold cover of $X$ branched along $Y$. We know $H_{*}\left(S^{3}-K\right) \rightarrow H_{*}\left(B^{4}-\Delta\right)$ is an isomorphism but generally, $H_{*}\left(\left(S^{3}-K\right)^{d}\right) \rightarrow H_{*}\left(\left(B^{4}-\Delta\right)^{d}\right)$ is not. It is true when $K$ is a ribbon knot: 


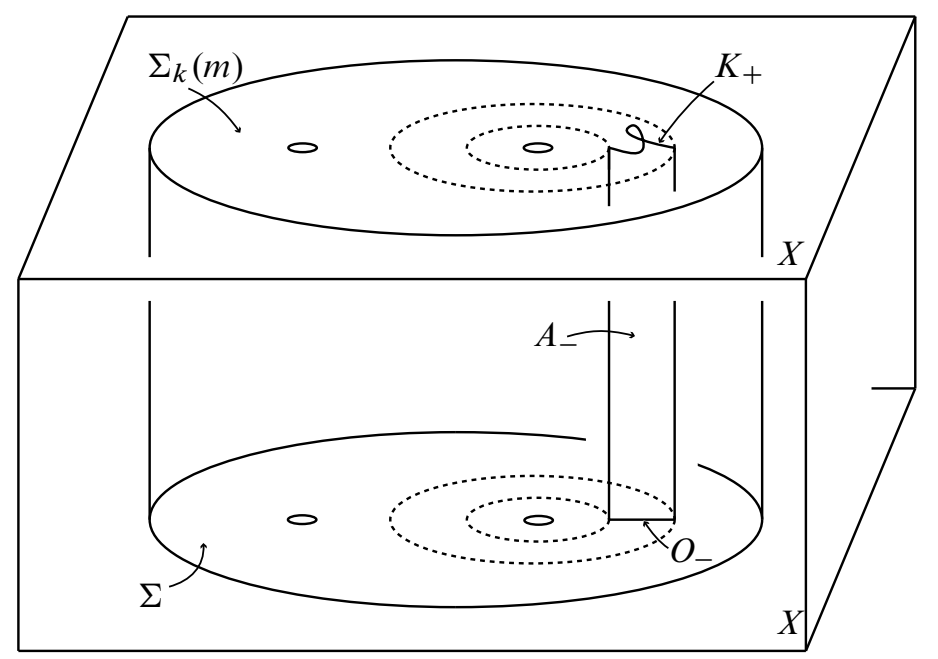

Figure 7: A cobordism between $(X, \Sigma)$ and $\left(X, \Sigma_{K}(m)\right)$

Lemma 4.2 If $K$ is a ribbon knot and the homology of $d$-fold cover of $S^{3}-K$, $H_{1}\left(\left(S^{3}-K\right)^{d}\right)$ is isomorphic to $\mathbb{Z}$ then the $d$-fold cover $\left(B^{4}-\Delta\right)^{d}$ of $B^{4}-\Delta$ is a homology circle.

Proof Let $\left(S^{3}-K\right)^{d}$ and $\left(B^{4}-\Delta\right)^{d}$ be the $d$-fold covers of $\left(S^{3}-K\right)$ and $\left(B^{4}-\Delta\right)$ according to the following homomorphisms $\varphi_{1}, \varphi_{2}$ :

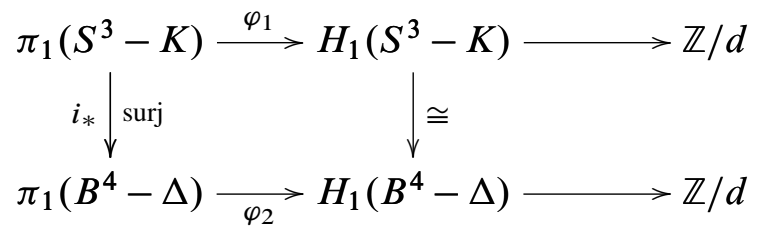

Since $K$ is a ribbon knot, $i_{*}: \pi_{1}\left(S^{3}-K\right) \rightarrow \pi_{1}\left(B^{4}-\Delta\right)$ is surjective. It follows that the map $H_{1}\left(\left(S^{3}-K\right)^{d}\right) \rightarrow H_{1}\left(\left(B^{4}-\Delta\right)^{d}\right)$ between the $d$-fold coverings is surjective since $i_{*}\left(\operatorname{ker} \varphi_{1}\right)$ maps to the trivial element of $\mathbb{Z} / d$ under $\varphi_{2}$. Since $H_{1}\left(\left(S^{3}-K\right)^{d}\right)$ is isomorphic to $\mathbb{Z}$, so is $H_{1}\left(\left(B^{4}-\Delta\right)^{d}\right)$. To show $H_{*}\left(\left(B^{4}-\Delta\right)^{d}\right)=0$ for $*>1$, we consider the long exact sequence of the pair $\left(\left(B^{4}-\Delta\right)^{d}, \partial\left(B^{4}-\Delta\right)^{d}\right)$.

$$
\begin{aligned}
& H_{4}\left(\left(B^{4}-\Delta\right)^{d}, \partial\left(B^{4}-\Delta\right)^{d}\right) \stackrel{\partial_{4}}{\rightarrow} H_{3}\left(\partial\left(B^{4}-\Delta\right)^{d}\right) \stackrel{i_{3}}{\rightarrow} H_{3}\left(B^{4}-\Delta\right)^{d} \\
& \quad \stackrel{j_{3}}{\rightarrow} H_{3}\left(\left(B^{4}-\Delta\right)^{d}, \partial\left(B^{4}-\Delta\right)^{d}\right) \stackrel{\partial_{3}}{\rightarrow} H_{2}\left(\partial\left(B^{4}-\Delta\right)^{d}\right) \stackrel{i_{2}}{\rightarrow} H_{2}\left(B^{4}-\Delta\right)^{d} \rightarrow \cdots
\end{aligned}
$$


Since $\partial_{4}$ is an isomorphism, $j_{3}$ is injective so that $H_{3}\left(\left(B^{4}-\Delta\right)^{d}\right)$ is isomorphic to im $j_{3}=\operatorname{ker} \partial_{3}$. Our claim is that $\partial_{3}: H_{3}\left(\left(B^{4}-\Delta\right)^{d}, \partial\left(B^{4}-\Delta\right)^{d}\right) \rightarrow H_{2}\left(\partial\left(B^{4}-\Delta\right)^{d}\right)$ is an isomorphism. Observe that $\partial\left(B^{4}-\Delta\right)^{d}=\left(S^{3}-K\right)^{d} \cup \widetilde{\Delta} \times \partial D^{2}$ where $\widetilde{\Delta}$ is the lifted disk of $\Delta$ in the $d$-fold cover of $B^{4}$. By Poincaré Duality and the Universal Coefficient Theorem,

$$
H_{3}\left(\left(B^{4}-\Delta\right)^{d}, \partial\left(B^{4}-\Delta\right)^{d}\right) \cong H^{1}\left(\left(B^{4}-\Delta\right)^{d}\right) \cong \operatorname{Hom}\left(H_{1}\left(\left(B^{4}-\Delta\right)^{d}\right), \mathbb{Z}\right)
$$

and

$$
\begin{aligned}
H_{2}\left(\left(S^{3}-K\right)^{d} \cup \widetilde{\Delta} \times \partial D^{2}\right) & \cong H^{1}\left(\left(S^{3}-K\right)^{d} \cup \widetilde{\Delta} \times \partial D^{2}\right) \\
& \cong \operatorname{Hom}\left(H_{1}\left(\left(S^{3}-K\right)^{d} \cup \widetilde{\Delta} \times \partial D^{2}\right), \mathbb{Z}\right)
\end{aligned}
$$

Since $H_{1}\left(\left(B^{4}-\Delta\right)^{d}\right)$ and $H_{1}\left(\left(S^{3}-K\right)^{d}\right)$ are isomorphic to the group $\mathbb{Z}$ generated by the lifted meridian $\tilde{\mu}$ of $K$ in $S^{3}$,

$$
H_{3}\left(\left(B^{4}-\Delta\right)^{d}, \partial\left(B^{4}-\Delta\right)^{d}\right) \cong H_{2}\left(\left(S^{3}-K\right)^{d} \cup \tilde{\Delta} \times \partial D^{2}\right) \cong \mathbb{Z}
$$

and moreover the boundary map $\partial_{3}$ induced by the restriction map from $\left(B^{4}-\Delta\right)^{d}$ to $\left(S^{3}-K\right)^{d}$. Hence $\partial_{3}$ is an isomorphism and so this proves that $H_{3}\left(\left(B^{4}-\Delta\right)^{d}\right)=0$ and also $H_{4}\left(\left(B^{4}-\Delta\right)^{d}\right)=0$.

Considering that the Euler characteristic of $\left(B^{4}-\Delta\right)^{d}$ is $\chi\left(B^{4}-\Delta\right)^{d}=d \cdot \chi\left(B^{4}-\Delta\right)$ and $H_{*}\left(S^{3}-K\right) \rightarrow H_{*}\left(B^{4}-\Delta\right)$ is an isomorphism, we get $H_{2}\left(\left(B^{4}-\Delta\right)^{d}\right)=0$.

Remark We may look at Example 4.6 to see infinitely many knots whose $d$-fold covers satisfy the condition in Lemma 4.2.

In the following Proposition, we will show that $W$ in Definition 4.1 is a homology cobordism. The condition that $K$ is a ribbon knot allows us to show that it is in fact a relative $h$-cobordism.

Proposition 4.3 If $K$ is a ribbon knot and the homology of $d$-fold cover $\left(S^{3}-K\right)^{d}$ of $S^{3}-K, H_{1}\left(\left(S^{3}-K\right)^{d}\right) \cong \mathbb{Z}$ with $d \equiv \pm 1(\bmod m)$ then there exists an $h$-cobordism $W$ between $M_{0}=X-\Sigma$ and $M_{1}=X-\Sigma_{K}(m)$ rel $\partial$.

Proof Keeping the previous notation in mind, let's denote $W=X \times I-(\Sigma \times I)_{A}(m)$, $M_{0}=X-\Sigma$ and $M_{1}=X-\Sigma_{K}(m)$. To show that $W$ is $H_{*}$-cobordism rel $\partial$, we'll prove $H_{*}\left(W, M_{1}\right)=H_{*}\left(W, M_{0}\right)=0$.

First, we need to describe $W$ and $M_{1}$ as follows; if we take a neighborhood of the curve $\alpha$ in $\Sigma$ as $S^{1} \times B^{3}$ meeting $\Sigma$ on $S^{1} \times I$ then denoting the complement of $S^{1} \times I$ in $\Sigma$ by $\Sigma_{0}$, we may write

$$
W=\left(X-S^{1} \times B^{3}-\Sigma_{0}\right) \times I \cup S^{1} \times_{\tau^{m}}\left(B^{3} \times I-A_{+}\right)
$$


and

$$
M_{1}=\left(X-S^{1} \times B^{3}-\Sigma_{0}\right) \cup S^{1} \times_{\tau_{K}^{m}}\left(B^{3}-K_{+}\right)
$$

Then considering the above description, the relative Mayer-Vietoris sequence shows

$$
H_{*}\left(W, M_{1}\right) \cong H_{*}\left(S^{1} \times_{\tau^{m}}\left(B^{3} \times I-A_{+}\right), S^{1} \times \tau_{K}^{m}\left(B^{3}-K_{+}\right)\right) .
$$

By the Alexander Duality, this relative homology group is same as

$$
H_{*}\left(S^{1} \times{ }_{\tau^{m}}\left(B^{3} \times I, A_{+}\right), S^{1} \times{ }_{\tau_{K}^{m}}\left(B^{3}, K_{+}\right)\right)
$$

which is trivial. Similarly, we can show that $H_{*}\left(W, M_{0}\right)$ is trivial as well.

A similar argument shows that $H_{*}\left(V, \partial M_{0}\right)$ is trivial and hence we have shown that $W$ is a homology cobordism from $M_{0}$ to $M_{1}$ rel $\partial$. To assert that $W$ is a relative $h$-cobordism, we need to show that $\pi_{1}(W)=\pi_{1}\left(X \times I-v(\Sigma \times I)_{A}(m)\right) \cong \mathbb{Z} / d$.

For simplicity let us denote $U=X-S^{1} \times B^{3}-\Sigma_{0}$ and $V=S^{1} \times \tau^{m}\left(B^{3}-K_{+}\right)$in the decomposition $\left(X-S^{1} \times B^{3}-\Sigma_{0}\right) \cup S^{1} \times{ }_{\tau^{m}}\left(B^{3}-K_{+}\right)$of $X-\Sigma_{K}(m)$. Then $U \cap V=S^{1} \times\left(\partial B^{3}-\{\right.$ two points $\left.\}\right)$. Denoting $V^{\prime}=S^{1} \times \tau^{m}\left(B^{3} \times I-A_{+}\right)$, we also rewrite

$$
W=\left(X-S^{1} \times B^{3}-\Sigma_{0}\right) \times I \cup S^{1} \times_{\tau^{m}}\left(B^{3} \times I-A_{+}\right)=U \times I \cup V^{\prime} .
$$

Then the intersection $U \times I \cap V^{\prime}$ is $S^{1} \times\left(\partial B^{3}-\{\right.$ two points $\left.\}\right) \times I=(U \cap V) \times I$. Applying Van Kampen's theorem for these decompositions of $M_{1}$ and $W$, we have the two commutative diagrams:

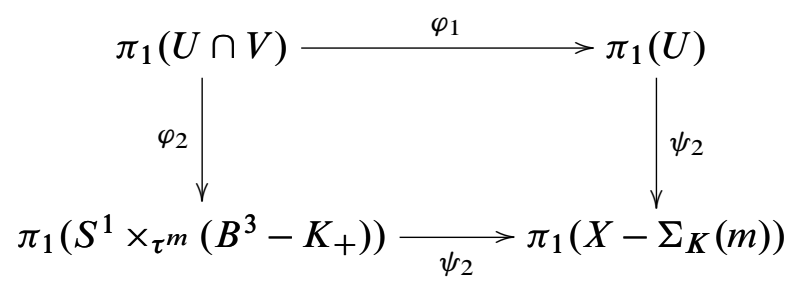

and

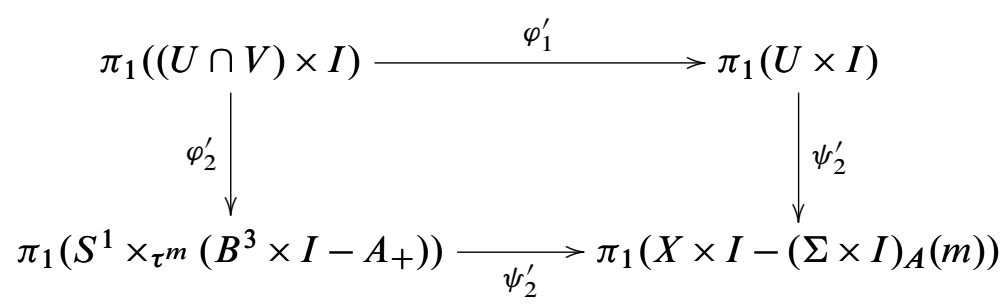


Let

$$
\begin{aligned}
& i_{1}: \pi_{1}(U \cap V) \rightarrow \pi_{1}((U \cap V) \times I) \\
& i_{2}: \pi_{1}(U) \rightarrow \pi_{1}(U \times I) \\
& i_{3}: \pi_{1}\left(S^{1} \times{ }_{\tau^{m}}\left(B^{3}-K_{+}\right)\right) \rightarrow \pi_{1}\left(S^{1} \times{ }_{\tau^{m}}\left(B^{3} \times I-A_{+}\right)\right)
\end{aligned}
$$

be the maps induced by inclusions. Then clearly $i_{1}$ and $i_{2}$ are isomorphisms. To show that $i_{3}$ is surjective, let's consider the fundamental group of mapping cylinders $S^{1} \times{ }_{\tau^{m}}\left(B^{3}-K_{+}\right)$and $S^{1} \times{ }_{\tau^{m}}\left(B^{3} \times I-A_{+}\right)$. Then representing the element $\left[S^{1}\right]$ in the fundamental group as $\alpha^{\prime}$, we present

$$
\begin{aligned}
& \pi_{1}\left(S^{1} \times \tau^{m}\left(B^{3}-K_{+}\right)\right)= \\
& \left.\quad\left\langle\pi_{1}\left(B^{3}-K_{+}\right), \alpha^{\prime}\right| \alpha^{\prime-1} \beta \alpha^{\prime}=\tau_{K *}^{m}(\beta) \text { for all } \beta \in \pi_{1}\left(B^{3}-K_{+}\right)\right\rangle .
\end{aligned}
$$

and

$$
\begin{aligned}
& \pi_{1}\left(S^{1} \times_{\tau^{m}}\left(B^{3} \times I-A_{+}\right)\right)= \\
& \left.\quad\left\langle\pi_{1}\left(B^{3} \times I-A_{+}\right), \alpha^{\prime}\right| \alpha^{\prime-1} \beta^{\prime} \alpha^{\prime}=\tau_{*}^{m}\left(\beta^{\prime}\right) \text { for all } \beta^{\prime} \in \pi_{1}\left(B^{3} \times I-A_{+}\right)\right\rangle .
\end{aligned}
$$

Since $K$ is a ribbon knot, $\pi_{1}\left(S^{3}-K\right) \longrightarrow \pi_{1}\left(S^{3} \times I-A\right)$ is surjective. So is $i_{3}$. Then by chasing the diagram, we have a surjective map

$$
\pi_{1}\left(X-\Sigma_{K}(m)\right) \rightarrow \pi_{1}\left(X \times I-(\Sigma \times I)_{A}(m)\right) .
$$

By Proposition 3.3, $\pi_{1}\left(X-\Sigma_{K}(m)\right)=\mathbb{Z} / d$. Since $W$ is an $H_{*}$-cobordism by the above argument, $H_{1}\left(X \times I-(\Sigma \times I)_{A}(m)\right)=\mathbb{Z} / d$ so that $\pi_{1}\left(X \times I-(\Sigma \times I)_{A}(m)\right)=$ $\mathbb{Z} / d$.

Now let us prove that the inclusion $i: M_{1} \longrightarrow W$ is a homotopy equivalence. The above work shows that the induced map $i_{*}: \pi_{1} M_{1} \longrightarrow \pi_{1} W \cong \mathbb{Z} / d$ is an isomorphism. So, the $d$-fold covers $W^{d}$ and $M_{1}{ }^{d}$ of $W$ and $M_{1}$ become universal covers and so we denote $\widetilde{W}=W^{d}, \widetilde{M}_{1}=M_{1}{ }^{d}$. Then we claim that the inclusion $\widetilde{M}_{1} \rightarrow \widetilde{W}$ induces an isomorphism in homology. Considering the decompositions of $W$ and $M_{1}$ in (2) and (3), we can express their $d$-fold covers as the $d$-fold covers of subcomponents associated to their inclusion maps to $H_{1}(W) \cong \mathbb{Z} / d$ :

$$
\begin{aligned}
\widetilde{W} & =\left(X \times I-(\Sigma \times I)_{A}(m)\right)^{d} \\
& =\left(\left(X-S^{1} \times B^{3}-\Sigma_{0}\right) \times I\right)^{d} \cup\left(S^{1} \times{ }_{\tau^{m}}\left(B^{3} \times I-A_{+}\right)\right)^{d}
\end{aligned}
$$

and

$$
\left.\widetilde{M}_{1}=\left(X-\Sigma_{K}(m)\right)\right)^{d}=\left(X-S^{1} \times B^{3}-\Sigma_{0}\right)^{d} \cup\left(S^{1} \times_{\tau_{K}^{m}}\left(B^{3}-K_{+}\right)\right)^{d} .
$$


In the inclusion-induced map $j: H_{1}\left(S^{1} \times{ }_{\tau^{m}}\left(B^{3} \times I-A_{+}\right)\right) \longrightarrow H_{1}(W) \cong \mathbb{Z} / d$, from our choice of the curve $\alpha$ in $\Sigma$ mentioned in the beginning of Section 3, we can easily check that in the Mayer-Vietoris sequence, the homology element $\left[S^{1} \times p t \times 0\right]$ with $p t \in\left(\partial B^{3}-\right.$ two points $)$ maps under $j$ to a trivial element in $H_{1}(W)$. The Mayer-Vietoris sequence for the decomposition of $W$ follows.

$$
\begin{aligned}
& \cdots \longrightarrow H_{1}\left(S^{1} \times\left(\partial B^{3}-\{\text { two points }\}\right) \times I\right) \stackrel{\varphi}{\longrightarrow} \\
& \quad \stackrel{\varphi}{\longrightarrow} H_{1}\left(\left(X-S^{1} \times B^{3}-\Sigma_{0}\right) \times I\right) \oplus H_{1}\left(S^{1} \times \tau^{m}\left(B^{3} \times I-A_{+}\right)\right) \stackrel{\psi}{\longrightarrow} \\
& \quad \stackrel{\psi}{\longrightarrow} H_{1}(W) \longrightarrow 0 .
\end{aligned}
$$

First we note the image of a generator $\left[S^{1} \times p t \times 0\right] \in H_{1}\left(S^{1} \times\left(\partial B^{3}-\{\right.\right.$ two points $\left.\left.\}\right) \times I\right)$ under $\varphi$ is $\left(0,\left[S^{1} \times p t \times 0\right]\right) \in H_{1}\left(\left(X-S^{1} \times B^{3}-\Sigma_{0}\right) \times I\right) \oplus H_{1}\left(S^{1} \times \tau^{m}\left(B^{3} \times I-A_{+}\right)\right)$ since the pushed-off curve of $\alpha$ along a trivialization is zero in $H_{1}\left(\left(X-S^{1} \times B^{3}-\Sigma_{0}\right) \cong\right.$ $H_{1}(X-\Sigma)$ by adjusting the framing of the curve $\alpha$.

So, since $\left(0,\left[S^{1} \times p t \times 0\right]\right)$ is in the kernel of $\psi,\left[S^{1} \times p t \times 0\right]$ maps to the trivial element in $H_{1}(W)$. Then we know the $d$-fold cover of $S^{1} \times{ }_{\tau^{m}}\left(B^{3} \times I-A_{+}\right)$has the form

$$
S^{1} \times \widetilde{\tau}^{m}\left(B^{3} \times I-A_{+}\right)^{d}
$$

for a proper lifted map $\tilde{\tau}^{m}$ of $\tau^{m}$ and by the same reason, the $d$-fold cover of

$$
S^{1} \times_{\tau_{K}^{m}}\left(B^{3}-K_{+}\right)
$$

is also of the form

$$
S^{1} \times \tilde{\tau}_{K}^{m}\left(B^{3}-K_{+}\right)^{d}
$$

for some lift $\tilde{\tau}_{K}^{m}$ of $\tau_{K}^{m}$.

Then we have a simple form of the relative homology of the pair $\left(W, M_{1}\right)$,

$$
H_{*}\left(\widetilde{W}, \widetilde{M}_{1}\right) \cong H_{*}\left(S^{1} \times_{\widetilde{\tau}^{m}}\left(B^{3} \times I-A_{+}\right)^{d}, S^{1} \times_{\widetilde{\tau}_{K}^{m}}\left(B^{3}-K_{+}\right)^{d}\right) .
$$

Since $K$ is a ribbon knot and $H_{1}\left(\left(S^{3}-K\right)^{d}\right) \cong \mathbb{Z}$, it follows by Lemma 4.2 that $H_{*}\left(\left(B^{3} \times I-A_{+}\right)^{d},\left(B^{3}-K_{+}\right)^{d}\right)=0$. So, the homology $H_{*}\left(\widetilde{W}, \widetilde{M}_{1}\right)$ is trivial. By the Whitehead theorem, we get $\pi_{n} \widetilde{M}_{1} \cong \pi_{n} \widetilde{W}$ for $n>1$. Since $\pi_{n} \widetilde{M}_{1} \cong \pi_{n} M_{1}$ and $\pi_{n} \widetilde{W} \cong \pi_{n} W$, it follows that $i_{*}: \pi_{n} M_{1} \rightarrow \pi_{n} W$ is an isomorphism. Therefore, again by Whitehead's theorem, $i: M_{1} \longrightarrow W$ is a homotopy equivalence.

Now we need to recall the definition of torsion, as given in [11] or [15] to show the Whitehead torsion of the pair $\left(W, M_{0}\right)$ constructed above is zero. 
Let $\Lambda$ be an associative ring with unit such that for any $r \neq s \in \mathbb{N}, \Lambda^{r}$ and $\Lambda^{s}$ are not isomorphic as $\Lambda$-modules. Consider an acyclic chain complex $C$ of length $m$ over $\Lambda$ whose chain groups are finite free $\Lambda$-modules with a preferred basis $c_{i}$ for each chain complex $C_{i}$. Then the torsion of the chain complex $C-$ written $\tau(C)-$ is defined as follows.

Let $G L(\Lambda)=\bigcup_{n \geq 0} G L(n, \Lambda)$ be the infinite general group. The torsion $\tau(C)$ will be an element of the abelianization of $G L(\Lambda)$, denoted by $K_{1}(\Lambda)$. Pick ordered bases $b_{i}$ of $B_{i}=\operatorname{Im} \partial_{i}$ and combine them to bases $b_{i} b_{i-1}$ of $C_{i}$. For the distinguished basis $c_{i}$ of $C_{i}$, let $\left(b_{i} b_{i-1} / c_{i}\right)$ is the transition matrix over $\Lambda$. Denoting the corresponding element of $K_{1}(\Lambda)$ by $\left[b_{i} b_{i-1} / c_{i}\right]$, define the torsion

$$
\tau(C)=\prod_{i=0}^{m}\left[b_{i} b_{i-1} / c_{i}\right]^{(-1)^{i+1}} \in K_{1}(\Lambda) .
$$

In particular, if $(K, L)$ is a pair of finite, connected CW complexes such that $L$ is a deformation retract of $K$ then consider the universal covering complexes $\widetilde{K} \supset \widetilde{L}$ of $K$ and $L$. Let's denote $\pi$ by the fundamental group of $K$. Then we obtain an acyclic free chain $\mathbb{Z}[\pi]$-complex $C(\widetilde{K}, \widetilde{L})$. So we have a well defined torsion $\tau=\tau(K, L)$ in the Whitehead group $\mathrm{Wh}(\pi)=K_{1}(\mathbb{Z}[\pi]) / \pm \pi$, the so-called 'Whitehead torsion'.

The $h$-cobordism we have constructed is built out of several pieces, and so our strategy is to compute the Whitehead torsion in terms of those pieces. The pieces may not be $h$-cobordisms, so they don't have a well-defined Whitehead torsion. However, they do have a more general kind of torsion, the Reidemeister-Franz torsion, which we briefly outline. It will turn out that the Reidemeister-Franz torsion of the pieces determines the Whitehead torsion of the $h$-cobordism. Moreover, the Reidemeister-Franz torsion satisfies gluing laws which will be able us to compute its value in terms of the pieces.

The 'Reidemeister-Franz torsion' is defined as follows. Consider the pair $(K, L)$ of finite, connected $\mathrm{CW}$-complexes but not requiring that $L$ is a deformation retract of $K$. Then keeping the notation above, the cellular chain group $C_{i}(\widetilde{K}, \widetilde{L})$ is a free $\mathbb{Z}[\pi]$-module as before. Let $\Lambda$ be an associative ring with unit with the above property. Given a ring homomorphism $\varphi: \mathbb{Z}[\pi] \longrightarrow \Lambda$, consider a free chain complex

$$
C^{\varphi}(K, L)=\Lambda \otimes_{\varphi} C(\widetilde{K}, \widetilde{L})
$$

If $C^{\varphi}$ is acyclic, the torsion corresponding the chain complex $C^{\varphi}$ is well defined. We will denote $\tau^{\varphi}(K, L) \in K_{1}(\Lambda) / \pm \varphi(\pi)$. If $\Lambda$ is a field then $K_{1}(\Lambda)=\Lambda^{*}$ so that $\tau^{\varphi}(K, L) \in \Lambda^{*} / \pm \varphi(\pi)$.

If the original complex $C$ is acyclic then the new complex $C^{\varphi}$ is also acyclic and so when the Whitehead torsion of $(K, L)$ is defined, the Reidemeister torsion of $(K, L)$ 
is also defined associated to the identity homomorphism id: $\mathbb{Z}[\pi] \longrightarrow \mathbb{Z}[\pi]$. However the relation

$$
\tau^{\varphi}(K, L)=\varphi_{*} \tau(K, L)
$$

shows that if the Reidemeister torsion associated to the identity is trivial then the Whitehead torsion is zero. We also need to know some formulas to compute torsion. Suppose $K=K_{1} \cup K_{2}, K_{0}=K_{1} \cap K_{2}, L=L_{1} \cup L_{2}, L_{0}=L_{1} \cap L_{2}$ and that $i: L \longrightarrow K$ is the inclusion which is restricted to homotopy equivalences $i_{\alpha}: L_{\alpha} \longrightarrow K_{\alpha}$ (for $\alpha=0,1,2$ ). Then $i$ is a homotopy equivalence and we have a formula called the 'sum theorem' in Whitehead torsion (see [15])

$$
\tau(K, L)=i_{1 *} \tau\left(K_{1}, L_{1}\right)+i_{2 *} \tau\left(K_{2}, L_{2}\right)-i_{0 *} \tau\left(K_{0}, L_{0}\right) .
$$

Using the multiplicativity of the torsion and the Mayer-Vietoris sequence we obtain a similar one called the 'gluing formula' in the Reidemeister torsion (see [15]).

Given subcomplexes $X_{1}$ and $X_{2}$ of $X$ such that $X=X_{1} \cup X_{2}$ and $X_{1} \cap X_{2}=$ $Y$, let $\varphi: \mathbb{Z}\left[H_{1}(X)\right] \longrightarrow \Lambda$ be a ring morphism where $\Lambda$ is a ring as above. Let $i: \mathbb{Z}\left[H_{1}(Y)\right] \longrightarrow \mathbb{Z}\left[H_{1}(X)\right]$ and $i_{\alpha}: \mathbb{Z}\left[H_{1}\left(X_{\alpha}\right)\right] \longrightarrow \mathbb{Z}\left[H_{1}(X)\right]$ (for $\alpha=1,2$ ) denote the inclusion-induced morphisms. If $\tau^{\varphi \circ i}(Y) \neq 0$ then we have the gluing formula

$$
\tau^{\varphi}(X) \cdot \tau^{\varphi \circ i}(Y)=\tau^{\varphi \circ i_{1}}\left(X_{1}\right) \cdot \tau^{\varphi \circ i_{2}}\left(X_{2}\right) .
$$

Now considering our situation, we have shown that $W$ is a relative $h$-cobordism from $M_{0}$ to $M_{1}$ with $\pi_{1}(W) \cong \mathbb{Z} / d$ and so the Whitehead torsion $\tau\left(W, M_{0}\right) \in W h(\mathbb{Z} / d)$ is defined. Recall that the decomposition of the pair

$$
\left(W, M_{0}\right)=\left(X \times I-(\Sigma \times I)_{A}(m), X-\Sigma\right)
$$

in (2) and (3) is

$$
\left(\left(X-S^{1} \times B^{3}-\Sigma_{0}\right) \times I \cup S^{1} \times_{\tau^{m}}\left(B^{3} \times I-A_{+}\right), X-S^{1} \times B^{3}-\Sigma_{0} \cup S^{1} \times\left(B^{3}-I\right)\right) .
$$

If we rewrite this as

$$
\left(\left(X-S^{1} \times B^{3}-\Sigma_{0}\right) \times I, X-S^{1} \times B^{3}-\Sigma_{0}\right) \cup\left(S^{1} \times \tau^{m}\left(B^{3} \times I-A_{+}\right), S^{1} \times\left(B^{3}-I\right)\right),
$$

then we can observe that the Whitehead torsion of the first component pair

$$
\left(\left(X-S^{1} \times B^{3}-\Sigma_{0}\right) \times I, X-S^{1} \times B^{3}-\Sigma_{0}\right)
$$

is zero and so we would like to attempt to use the sum theorem for this decomposition. But in the second pair, $S^{1} \times{ }_{\tau^{m}}\left(B^{3} \times I-A_{+}\right)$is just a homology cobordism which means $S^{1} \times\left(B^{3}-I\right)$ may not be a deformation retract of $S^{1} \times \tau^{m}\left(B^{3} \times I-A_{+}\right)$. Then the Whitehead torsion $\tau\left(S^{1} \times \tau^{m}\left(B^{3} \times I-A_{+}\right), S^{1} \times\left(B^{3}-I\right)\right)$ is not defined and thus we can not apply the sum theorem in order to show the Whitehead torsion $\tau\left(W, M_{0}\right)=0$. 
But we will show later that $\tau\left(S^{1} \times{ }_{\tau^{m}}\left(B^{3} \times I-A_{+}\right), S^{1} \times\left(B^{3}-I\right)\right)$ is well defined under an additional assumption to make the complex of the $d$-fold cover of the pair, $C\left(\left(S^{1} \times \tau^{m}\left(B^{3} \times I-A_{+}\right)\right)^{d},\left(S^{1} \times\left(B^{3}-I\right)\right)^{d}\right)$, acyclic with $\mathbb{Z}[\mathbb{Z} / d]$ coefficient. So instead of computing the Whitehead torsion, we will show that the Reidemeister torsion $\tau^{\text {id }}\left(W, M_{0}\right)$, denoted simply by $\tau\left(W, M_{0}\right)$, according to the coefficient $\mathbb{Z}[\mathbb{Z} / d]$ is trivial. Applying the gluing formula to the above decomposition instead of the sum theorem, we can obtain a simpler method to compute the Reidemeister torsion for the pair $\left(W, M_{0}\right)$.

Now we first need to consider the torsion of certain fibration over a circle with a homologically trivial fiber.

A relative fiber bundle

$$
\left(F, F_{0}\right) \hookrightarrow(X, Y) \stackrel{\pi}{\longrightarrow} S^{1}
$$

means that $F \hookrightarrow X \stackrel{\pi}{\longrightarrow} S^{1}$ is a fiber bundle with a trivialization $\left\{\varphi_{\alpha}, U_{\alpha}\right\}$ satisfying that for an open cover $U_{\alpha} \subset S^{1},\left(\pi^{-1}(U), Y \cap \pi^{-1}(U)\right) \cong U \times\left(F, F_{0}\right)$ and the diagram

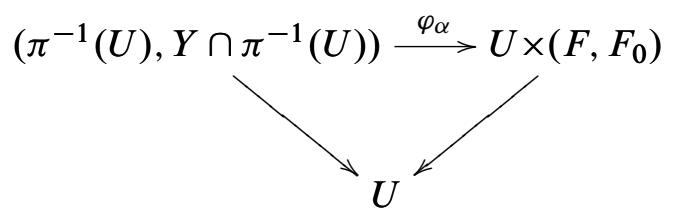

commutes. We will now prove the following result.

Proposition 4.4 Let $\left(F, F_{0}\right) \hookrightarrow(X, Y) \longrightarrow S^{1}$ be a smooth, relative fiber bundle over $S^{1}$ such that the fiber pair $\left(F, F_{0}\right)$ is homologically trivial. Suppose that $G$ is a group and $\rho: H_{1}(X) \longrightarrow G$ is a group homomorphism such that the image under $\rho$ of the homology class $\left[S^{1}\right] \in H_{1}(X)$ of the base space in the fibration has finite order in $G$. Let $\left(\widetilde{F}, \widetilde{F}_{0}\right)$ be the cover of $\left(F, F_{0}\right)$ associated to the homomorphism

$$
H_{1}(F) \hookrightarrow H_{1}(X) \stackrel{\rho}{\longrightarrow} G
$$

and denote again by $\rho$ the induced map $\mathbb{Z}\left[H_{1}(X)\right] \longrightarrow \mathbb{Z}[G]$. If the cover $\left(\widetilde{F}, \widetilde{F}_{0}\right)$ is homologically trivial, that is $H_{*}\left(F, F_{0} ; \mathbb{Z}[G]\right)=0$ then the torsion $\tau^{\rho}(X, Y) \in$ $K_{1}(\mathbb{Z}[G]) / \pm G$ is trivial.

Proof We may assume that $X$ is a mapping torus $X=S^{1} \times{ }_{\varphi} F$ with the monodromy map $\varphi$ of the fibration. Let $(\widetilde{X}, \widetilde{Y})$ be the cover of $(X, Y)$ associated to $\rho$. Then $\widetilde{X}$ is also a mapping torus since the homology image $\rho\left(\left[S^{1}\right]\right)$ is of finite order in $G$. So, let us say $\widetilde{X}=S^{1} \times \widetilde{\varphi} \widetilde{F}$ where $\widetilde{F}$ is the cover associated to $H_{1}(F) \hookrightarrow H_{1}(X) \stackrel{\rho}{\longrightarrow} G$ 
and $\tilde{\varphi}$ is a lift of $\varphi$ in $\widetilde{X}$. Similarly, we also say $\widetilde{Y}=S^{1} \times \widetilde{\varphi} \widetilde{F}_{0}$. Considering the Wang exact homology sequence and the Five Lemma we have

$$
\begin{aligned}
& H_{*}\left(\widetilde{F}_{0}\right) \stackrel{\widetilde{\varphi}_{*}^{-1}}{\longrightarrow} H_{*}\left(\widetilde{F}_{0}\right) \longrightarrow H_{*}\left(S^{1} \times \widetilde{\varphi} \widetilde{F}_{0}\right) \longrightarrow H_{*-1}\left(\widetilde{F}_{0}\right) \stackrel{\widetilde{\varphi}_{*}^{-1}}{\longrightarrow} H_{*-1}\left(\widetilde{F}_{0}\right) \\
& \cong \downarrow \quad \cong \downarrow \quad \_\downarrow \quad \cong \downarrow \\
& H_{*}(\widetilde{F}) \stackrel{\widetilde{\varphi}_{*}^{-1}}{\longrightarrow} H_{*}(\widetilde{F}) \longrightarrow H_{*}\left(S^{1} \times_{\widetilde{\varphi}} \widetilde{F}\right) \longrightarrow H_{*-1}(\widetilde{F}) \stackrel{\widetilde{\varphi}_{*}^{-1}}{\longrightarrow} H_{*-1}(\widetilde{F})
\end{aligned}
$$

and we get an acyclic complex $C_{*}\left(S^{1} \times \widetilde{\varphi} \widetilde{F}, S^{1} \times_{\widetilde{\varphi}} \widetilde{F}_{0}\right)$ since $H_{*}\left(\widetilde{F}_{0}\right) \longrightarrow H_{*}(\widetilde{F})$ is an isomorphism. Thus, the associated torsion $\tau^{\rho}(X, Y)$ is defined.

Now we consider the Mayer-Vietoris sequence for $(\widetilde{X}, \widetilde{Y})=\left(S^{1} \times \widetilde{\varphi} \widetilde{F}, S^{1} \times \widetilde{\varphi} \widetilde{F}_{0}\right)$. Let us consider closed manifold pairs $\left(X_{1}, Y_{1}\right)=\left(\left[0, \frac{1}{2}\right] \times \widetilde{F},\left[0, \frac{1}{2}\right] \times \widetilde{F}_{0}\right)$ and $\left(X_{2}, Y_{2}\right)=$ $\left(\left[\frac{1}{2}, 1\right] \times \widetilde{F},\left[\frac{1}{2}, 1\right] \times \widetilde{F}_{0}\right)$. Define a map $f$ of a subspace $A:=\{0\} \times \widetilde{F} \cup\left\{\frac{1}{2}\right\} \times \widetilde{F}$ of $X_{1}$ into $X_{2}$ by

$$
\left.f\right|_{\{0\} \times \widetilde{F}}=\widetilde{\varphi} \times\{1\},\left.f\right|_{\{1 / 2\} \times \widetilde{F}}=1_{\{1 / 2\} \times \widetilde{F}} .
$$

Then letting $B:=\{0\} \times \widetilde{F}_{0} \cup\left\{\frac{1}{2}\right\} \times \widetilde{F}_{0} \subset A$, we can consider $\left(S^{1} \times \widetilde{\varphi} \widetilde{F}, S^{1} \times \widetilde{\varphi} \widetilde{F}_{0}\right)$ as the adjunction space $\left(X_{1} \cup_{f} X_{2}, Y_{1} \cup_{f} Y_{2}\right)$ of the system $\left(X_{1}, Y_{1}\right) \supset(A, B) \stackrel{f}{\longrightarrow}\left(X_{2}, Y_{2}\right)$. There is a short exact sequence

$$
\begin{aligned}
0 \longrightarrow C_{*}\left(X_{1} \cap X_{2}, Y_{1} \cap Y_{2}\right) & \longrightarrow C_{*}\left(X_{1}, Y_{1}\right) \oplus C_{*}\left(X_{2}, Y_{2}\right) \\
& \longrightarrow C_{*}\left(X_{1} \cup_{f} X_{2}, Y_{1} \cup_{f} Y_{2}\right) \longrightarrow 0 .
\end{aligned}
$$

If we rewrite this then we have

$$
\begin{aligned}
\left.0 \longrightarrow C_{*}\left(\widetilde{F}, \widetilde{F}_{0}\right) \oplus C_{*}\left(\widetilde{F}, \widetilde{F}_{0}\right)\right) & \\
& \longrightarrow C_{*}\left([0,1 / 2] \times \widetilde{F},[0,1 / 2] \times \widetilde{F}_{0}\right) \oplus C_{*}\left([1 / 2,1] \times \widetilde{F},[1 / 2,1] \times \widetilde{F}_{0}\right) \\
& \longrightarrow C_{*}\left(S^{1} \times \widetilde{\varphi} \widetilde{F}, S^{1} \times \widetilde{\varphi} \widetilde{F}_{0}\right) \longrightarrow 0 .
\end{aligned}
$$

If $\left(\widetilde{F}, \widetilde{F}_{0}\right)$ is homologically trivial, it follows that if $j: \mathbb{Z}\left[H_{1}(F)\right] \longrightarrow \mathbb{Z}\left[H_{1}(X)\right]$ denotes the morphism induced by inclusion then the torsion $\tau^{\rho \circ j}\left(F, F_{0}\right)$ is defined. From the above short exact sequence and the multiplicativity of the torsion we deduce that

$$
\tau^{\rho \circ j}\left(F, F_{0}\right) \cdot \tau^{\rho \circ j}\left(F, F_{0}\right)=\left(\tau^{\rho \circ j}\left(F, F_{0}\right) \cdot \tau^{\rho \circ j}\left(F, F_{0}\right)\right) \cdot \tau^{\rho}\left(S^{1} \times_{\varphi} F, S^{1} \times_{\varphi} F_{0}\right) .
$$

This implies that $\tau^{\rho}\left(S^{1} \times_{\varphi} F, S^{1} \times_{\varphi} F_{0}\right)=\tau^{\rho}(X, Y) \in K_{1}(\mathbb{Z}[G]) / \pm G$ is trivial.

Using the proposition above, we get topological equivalence classes of $\left(X, \Sigma_{K}(m)\right)$ under the following condition. 
Theorem 4.5 If $K$ is a ribbon knot and the homology of $d$-fold cover $\left(S^{3}-K\right)^{d}$ of $S^{3}-K, H_{1}\left(\left(S^{3}-K\right)^{d}\right) \cong \mathbb{Z}$ with $d \equiv \pm 1(\bmod m)$ then $(X, \Sigma)$ is pairwise homeomorphic to $\left(X, \Sigma_{K}(m)\right)$.

Proof Under these assumptions, we have a relative $h$-cobordism $W$ from $M_{0}=$ $X-\Sigma$ to $M_{1}=X-\Sigma_{K}(m)$ by Proposition 4.3. As we discussed before, in order to show the Whitehead torsion $\tau\left(W, M_{0}\right)=0 \in W h(\mathbb{Z} / d)$, it is sufficient to show that the Reidemeister torsion $\tau\left(W, M_{0}\right) \in W h(\mathbb{Z} / d)$ associated to the identity map id: $\mathbb{Z}[\mathbb{Z} / d] \longrightarrow \mathbb{Z}[\mathbb{Z} / d]$ is trivial.

Consider the decomposition of the pair $\left(W, M_{0}\right)$,

$\left(\left(X-S^{1} \times B^{3}-\Sigma_{0}\right) \times I, X-S^{1} \times B^{3}-\Sigma_{0}\right) \cup\left(S^{1} \times \tau^{m}\left(B^{3} \times I-A_{+}\right), S^{1} \times\left(B^{3}-I\right)\right)$.

To apply the gluing formula of the Reidemeister torsion for this decomposition, we need to check the torsion of each component is defined.

First, the torsion $\tau\left(\left(X-S^{1} \times B^{3}-\Sigma_{0}\right) \times I, X-S^{1} \times B^{3}-\Sigma_{0}\right)$ is clearly defined and trivial. To check the torsion of the second component, we will show the relative chain complex $C\left(\left(S^{1} \times \tau^{m}\left(B^{3} \times I-A_{+}\right)\right)^{d},\left(S^{1} \times\left(B^{3}-I\right)\right)^{d}\right)$ of $d$-fold covers is acyclic.

The same argument in the proof of Proposition 4.3 shows that the $d$-fold cover

$$
\left(S^{1} \times \tau^{m}\left(B^{3} \times I-A_{+}\right)\right)^{d}
$$

associated to the inclusion-induced map

$$
j: H_{1}\left(S^{1} \times \tau^{m}\left(B^{3} \times I-A_{+}\right)\right) \longrightarrow H_{1}(W) \cong \mathbb{Z} / d
$$

is a mapping torus with the $d$-fold cover of $B^{3} \times I-A_{+}$that is $S^{1} \times \tilde{\tau}^{m}\left(B^{3} \times I-A_{+}\right)^{d}$. Similarly, the $d$-fold cover $\left(S^{1} \times{ }_{\tau^{m}}\left(B^{3}-I\right)\right)^{d}$ is $S^{1} \times \tilde{\tau}^{m}\left(B^{3}-I\right)^{d}$.

Observing the proof of Lemma 4.2, we have an isomorphism $H_{*}\left(\left(S^{3}-K\right)^{d}\right) \longrightarrow$ $H_{*}\left(\left(B^{4}-\Delta\right)^{d}\right)$ when $K$ is a ribbon knot and $H_{1}\left(\left(S^{3}-K\right)^{d}\right) \cong \mathbb{Z}$. In other words, $H_{*}\left(\left(B^{4}-\Delta\right)^{d},\left(S^{3}-K\right)^{d}\right)=0$. Excision argument shows that this is isomorphic to

$$
H_{*}\left(\left(B^{3} \times I-A_{+}\right)^{d},\left(B^{3}-K_{+}\right)^{d}\right)=0 \cong H_{*}\left(\left(B^{3} \times I-A_{+}\right)^{d},\left(B^{3}-I\right)^{d}\right) .
$$

This gives that

$$
\begin{aligned}
& H_{*}\left(\left(S^{1} \times \tau^{m}\left(B^{3} \times I-A_{+}\right)\right)^{d},\left(S^{1} \times \tau^{m}\left(B^{3}-I\right)\right)^{d}\right) \\
& \quad=H_{*}\left(S^{1} \times \tilde{\tau}^{m}\left(B^{3} \times I-A_{+}\right)^{d}, S^{1} \times \tilde{\tau}^{m}\left(B^{3}-I\right)^{d}\right)=0 .
\end{aligned}
$$


Then the torsion $\tau^{j}\left(S^{1} \times{ }_{\tau^{m}}\left(B^{3} \times I-A_{+}\right), S^{1} \times{ }_{\tau^{m}}\left(B^{3}-I\right)\right)$ associated to the induced ring homomorphism $j: \mathbb{Z}\left[H_{1}\left(S^{1} \times{ }_{\tau^{m}}\left(B^{3} \times I-A_{+}\right)\right)\right] \longrightarrow \mathbb{Z}\left[H_{1}(W)\right] \cong \mathbb{Z}[\mathbb{Z} / d]$ is defined.

Now applying the gluing formula of the Reidemeister torsion for the decomposition of $\left(W, M_{0}\right)$, we have

$$
\begin{gathered}
\tau\left(W, M_{0}\right) \cdot \tau\left(\partial\left(X-S^{1} \times B^{3}-\Sigma_{0}\right) \times I, \partial\left(X-S^{1} \times B^{3}-\Sigma_{0}\right)\right) \\
=\tau\left(\left(X-S^{1} \times B^{3}-\Sigma_{0}\right) \times I, X-S^{1} \times B^{3}-\Sigma_{0}\right) . \\
\tau\left(S^{1} \times{ }_{\tau^{m}}\left(B^{3} \times I-A_{+}\right), S^{1} \times\left(B^{3}-I\right)\right) .
\end{gathered}
$$

Hence,

$$
\tau\left(W, M_{0}\right)=\tau\left(S^{1} \times \tau^{m}\left(B^{3} \times I-A_{+}\right), S^{1} \times\left(B^{3}-I\right)\right) .
$$

To compute $\tau\left(S^{1} \times \tau^{m}\left(B^{3} \times I-A_{+}\right), S^{1} \times\left(B^{3}-I\right)\right)$, we note that

$$
\left(B^{3} \times I-A_{+}, B^{3}-I\right) \hookrightarrow\left(S^{1} \times{ }_{\tau^{m}}\left(B^{3} \times I-A_{+}\right), S^{1} \times\left(B^{3}-I\right)\right) \longrightarrow S^{1}
$$

is a smooth fiber bundle over $S^{1}$ with the fiber $\left(B^{3} \times I-A_{+}, B^{3}-I\right)$. Clearly the fiber $\left(B^{3} \times I-A_{+}, B^{3}-I\right)$ is homologically trivial and by the above argument, the $d$-fold cover $\left(\left(B^{3} \times I-A_{+}\right)^{d},\left(B^{3}-I\right)^{d}\right)$ associated to $j$ is also homologically trivial. Thus, by Proposition 4.4 the torsion $\tau\left(S^{1} \times \tau^{m}\left(B^{3} \times I-A_{+}\right), S^{1} \times\left(B^{3}-I\right)\right)$ is trivial and thus the Whitehead torsion $\tau\left(W, M_{0}\right)=0$. Then by Freedman's work [6], the $h$-cobordism $W$ is topologically trivial and so the complements $X-\Sigma$ and $X-\Sigma_{K}(m)$ are homeomorphic. The homeomorphism $\partial v(\Sigma) \longrightarrow \partial v\left(\Sigma_{K}(m)\right)$ extends to a homeomorphism $(X, \Sigma) \longrightarrow\left(X, \Sigma_{K}(m)\right)$.

Example 4.6 Let's consider examples $\left(X, \Sigma_{K}(m)\right)$ which are smoothly knotted but topologically standard. Let $J$ be a torus knot $T_{p, q}$ in $S^{3}$ such that $p$ and $q$ are coprime positive integers. Then we have a ribbon knot $K=J \#-J$ with its Alexander polynomial $\Delta_{K}(t)=\left(\Delta_{J}(t)\right)^{2}$ where

$$
\Delta_{J}(t)=\frac{(1-t)\left(1-t^{p q}\right)}{\left(1-t^{p}\right)\left(1-t^{q}\right)}
$$

Note that the $d$-fold cover of $S^{3}$ branched along the torus knot $J=T_{p, q}$ is the Brieskorn manifold $\Sigma(p, q, d)$, and that this manifold is a homology sphere if p,q and $\mathrm{d}$ are pairwise relatively prime. Since $\left(S^{3}, K\right)^{d}$ is $\Sigma(p, q, d) \# \Sigma(p, q, d),\left(S^{3}, K\right)^{d}$ is an integral homology 3 -sphere. We might obtain a direct proof for this by computing the order of $H_{1}\left(\left(S^{3}, K\right)^{d}\right)$ of $d$-fold cover $\left(S^{3}, K\right)^{d}$ of $S^{3}$ branched over $K$. In 
fact, Fox [5] proved that

$$
\left|H_{1}\left(\left(S^{3}, K\right)^{d}\right)\right|=\prod_{i=0}^{d-1} \Delta_{K}\left(\zeta^{i}\right)
$$

where $\zeta$ is a primitive $d$ th root of unity. And it's easy to show that

$$
\prod_{i=0}^{d-1} \Delta_{K}\left(\zeta^{i}\right)=1
$$

So, we obtain a ribbon knot $K$ with $\Delta_{K}(t) \neq 1$ and the $d$-fold branch cover $\left(S^{3}, K\right)^{d}$ is a homology 3-sphere when $(p, d)=1$ and $(q, d)=1$. Then by Theorem 3.4 and Theorem 4.5 , we have infinitely many pairs $\left(X, \Sigma_{K}(m)\right)$ which are smoothly knotted but not topologically.

\section{Acknowledgements}

I thank the referee for pointing out a gap in the proof of one of main theorems and other helpful comments. I would also like to thank Fintushel and Stern for their note and I would like to express my sincere gratitude to my advisor Daniel Ruberman for his tremendous help and support.

\section{References}

[1] S Finashin, Knotting of algebraic curves in $\mathbb{C P}^{2}$, Topology 41 (2002) 47-55 MR1871240

[2] R Fintushel, RJ Stern, Surfaces in 4-manifolds: Addendum arXiv: math.GT/0511707

[3] R Fintushel, R J Stern, Surfaces in 4-manifolds, Math. Res. Lett. 4 (1997) 907-914 MR1492129

[4] R Fintushel, R J Stern, Knots, links, and 4-manifolds, Invent. Math. 134 (1998) 363-400 MR1650308

[5] R H Fox, Free differential calculus III: Subgroups, Ann. of Math. (2) 64 (1956) 407419 MR0095876

[6] M H Freedman, The topology of four-dimensional manifolds, J. Differential Geom. 17 (1982) 357-453 MR679066

[7] R E Gompf, A new construction of symplectic manifolds, Ann. of Math. (2) 142 (1995) 527-595 MR1356781 
[8] C M Gordon, Ribbon concordance of knots in the 3-sphere, Math. Ann. 257 (1981) 157-170 MR634459

[9] R Kirby, Problems in low-dimensional topology, from: "Geometric topology (Athens, GA, 1993)", AMS/IP Stud. Adv. Math. 2, Amer. Math. Soc., Providence, RI (1997) 35-473 MR1470751

[10] P Kronheimer, T Mrowka, Floer homology for Seiberg-Witten monopoles, in preparation

[11] J Milnor, Whitehead torsion, Bull. Amer. Math. Soc. 72 (1966) 358-426 MR0196736

[12] J Morgan, H Bass, The Smith conjecture, Pure and Applied Mathematics 112, Academic Press Inc., Orlando, FL (1984) MR758459

[13] C H Taubes, The Seiberg-Witten invariants and symplectic forms, Math. Res. Lett. 1 (1994) 809-822 MR1306023

[14] C H Taubes, The Seiberg-Witten invariants and 4-manifolds with essential tori, Geom. Topol. 5 (2001) 441-519 MR1833751

[15] V Turaev, Introduction to combinatorial torsions, Lectures in Mathematics ETH Zürich, Birkhäuser Verlag, Basel (2001) MR1809561

[16] S Vidussi, Seiberg-Witten invariants for manifolds diffeomorphic outside a circle, Proc. Amer. Math. Soc. 129 (2001) 2489-2496 MR1823936

[17] E C Zeeman, Twisting spun knots, Trans. Amer. Math. Soc. 115 (1965) 471-495 MR0195085

Department of Mathematics, McMaster University

Hamilton, Ontario L8S 4K1, Canada

hjkim@math.mcmaster.ca

Proposed: Ronald Fintushel

Received: 22 July 2004

Seconded: Peter Ozsváth, Ronald Stern

Revised: 29 November 2005

Geometry 83 Topology, Volume 10 (2006) 